\title{
A propósito del Gravetiense... El paso de cultura a tecnocomplejo: un caso ejemplar de pervivencia particularista
}

\section{Changing Perceptions of the Gravettian: from Culture to Technocomplex}

\author{
Paloma de la Peña Alonso \\ Grupo de investigación en Geografía Física de Alta Montaña, UCM \\ paloma.delapenya@gmail.com
}

Recibido: 28-10-2011

Aceptado: 13-02-2012

\begin{abstract}
RESUMEN
En este trabajo se realiza un recorrido historiográfico sobre los conceptos de Gravetiense y Perigordiense. Se pretende mostrar cómo ha cambiado la consideración de este fenómeno desde cultura a tecnocomplejo, resaltando que, sin embargo, las bases teóricas que provocaron dicho cambio apenas han sido modificadas. Asimismo, se realiza una llamada de atención sobre la presente complacencia por la perspectiva tecnológica que, paradójicamente y en contra de sus fundamentos, está restringiendo notablemente la comprensión de este lapso del Paleolitico Superior europeo y manteniéndolo dentro de la antigua concepción particularista. Esta situación parece provocada por la ausencia de reflexión en torno a los términos manejados en el estudio del Paleolítico Superior.
\end{abstract}

Palabras Clave: Cultura. Complejo. Tecnocomplejo. Particularismo. Perigordiense. Gravetiense.

\begin{abstract}
This article gives a historiographic overview of the evolution of the concept of Gravettian, showing how the perception of this phenomenon has changed. It was originally regarded as a culture, but is now considered to be a technocomplex, despite the fact that the overall theoretical base has hardly changed. The article also advocates caution with regard to the current popularity of the technological perspective, which, paradoxically, restricts the understanding of this period of the European Upper Paleolithic to the old sphere of particularism. This situation appears to be the result of a lack of precision as regards Upper Paleolithic terminology.
\end{abstract}

Key words: Culture. Complex. Technocomplex. Particularism. Perigordian. Gravettian.

Sumario 1. El Gravetiense como cultura paneuropea: de Siberia al Algarve. 2. Indagación historiográfica sobre la definición del Gravetiense y posteriores re-elaboraciones. 2.1. H. Breuil y D. Peyrony: la toma en consideración. 2.2. D.A.E. Garrod: la creación de la cultura Gravetiense. 2.3. La cuestión perigordiense. 2.4. G. Laplace: el Gravetiense como Complejo. 2.5. Algunas alternativas procesuales. 2.6. La escuela tecnológica. 3. Los pilares de la visión unitaria del Gravetiense. 4. De cultura a tecnocomplejo. 5. Conclusiones. 
Les préhistoriens sont souvent victimes de ce qu'on pourrait appeler «la conception instantanée» des industries. Elle consiste à raisonner en cercle, et à dire: Ceci est du Périgordien IV parce qu'il y a des gravettes (en faisant abstraction de tout le reste de l'outillage), et il y a des gravettes parce que c'est $d u$ Périgordien IV.

La question Périgordien, F. Bordes (1968).

\section{El Gravetiense como cultura Paneuropea: de Siberia al Algarve}

El Gravetiense se define como un fenómeno de amplio espectro (cultural, social, económico, técnico, simbólico, etc.) que afectó durante prácticamente diez mil años a buena parte de Eurasia. A lo largo de la historia de su investigación se ha identificado desde Siberia hasta el Algarve. Historiográficamente se concibió como una cultura por algunos de los principales investigadores del Paleolítico a comienzos del siglo XX dentro de la corriente particularista o historicista-cultural. Actualmente, se le describe como un "tecnocomplejo", fruto de la nueva corriente tecnológica dentro de los estudios de Paleolítico, a pesar de que todavía subyace la idea de identidad y de unidad cultural, comparándose sus evidencias materiales y extrapolándose sin problemas en regiones alejadas unas de otras dentro del continente euroasiático, incluso cuando sus facies o variantes se van multiplicando cada vez más. En definitiva, su concepción ideal es la de una supuesta cultura paneuropea, una especie de Cristianitas que acaeció durante un largo periodo del Paleolítico Superior.

Su consideración en los últimos años contrasta con el Auriñaciense, que suscita un constante debate al verse involucrado en la manida cuestión de la transición Paleolítico Medio-Paleolítico Superior. Así, aquel ha sido objeto hasta de debates teóricos en torno a los conceptos manejados en las denominaciones de sus distintas variantes (Bon, 2002; VVAA 2006) en contraposición al Gravetiense que raramente se cuestiona, si bien recientemente sus estudios se han revitalizado en el occidente europeo, especialmente en el ámbito francés y español.

La situación de abandono con respecto a las fases anteriores del Paleolítico Superior inicial es sorprendente ya que durante toda la segunda mitad del siglo XX se discutió su estatus en relación al Auriñaciense y, en el caso de Europa central y oriental, constituye una de las manifestaciones culturales más complejas en términos antropológicos, sólo equiparable a las posteriores evidencias mesolíticas (Svoboda 2000; Zbelevil 2005).
El objetivo de este trabajo es recordar el inicio de la definición de Gravetiense y describir su evolución desde entonces. Finalmente se pretende señalar que la moderna corriente tecnológica ha retomado su uso en el sentido de civilización o gran cultura, re-denominándolo como tecnocomplejo y dividiéndolo en múltiples subvariantes. Esta situación supone una enorme contradicción al promulgar terminológicamente una vuelta a lo unitario cuando se demuestra su variabilidad extrema precisamente desde dicha perspectiva tecno-tipológica. A partir de este ejemplo específico se quiere plantear una reflexión en torno a los conceptos de cultura, tecnocomplejo y período cronológico que frecuentemente se confunden o intercambian en los modernos estudios de Paleolítico. En definitiva, queremos emprender una llamada de atención sobre los conceptos manejados desde el que es, a nuestro juicio, un caso paradigmático.

\section{Indagación historiográfica sobre la definición del Gravetiense y posteriores re-elaboraciones}

\subsection{H. Breuil y D. Peyrony: la toma en consi- deración}

A comienzos del siglo XX acaece uno de los debates más interesantes de cara a la reconstrucción racional de nuestra disciplina: la denominada $\mathrm{Ba}-$ talla del Auriñaciense, que supuso el nacimiento de lo que ahora consideramos Paleolítico Superior inicial (Groenen 1994). H. Breuil retomó el antiguo periodo Auriñaciense — desechado o, mejor dicho, postergado previamente en la clasificación de G. Mortillet (1885) —, situándolo entre el Musteriense y el Solutrense (Breuil 1906, 1907, 1909, 1912) y abriendo un debate que acarreó importantes implicaciones para el estudio del Paleolítico Superior.

Breuil basó su argumentación en minuciosos estudios estratigráficos unidos al análisis tipológico de las industrias de varios yacimientos belgas y franceses. En uno de sus primeros escritos se refirió a dichas industrias como "presolutrenses" (1906) para posteriormente retomar el término "Auriñaciense" (Fig. 1), dentro del que diferenció tres periodos: Inferior (de la Punta de Chatelperron), Medio (de la Punta de Aurignac) y Superior (de la Punta de La Gravette) (Breuil 1912). Este último - que es el que nos interesa en este estudio-, se caracterizaba por largas hojas rectilíneas, buriles de variados tamaños, pointes à soie, esculturas en bulto redondo (Venus de Willendorf, Grimaldi, etc.), grabados (Laussel), etc.

La correcta situación estratigráfica del Auriñaciense permitió a Breuil definir el inicio del 


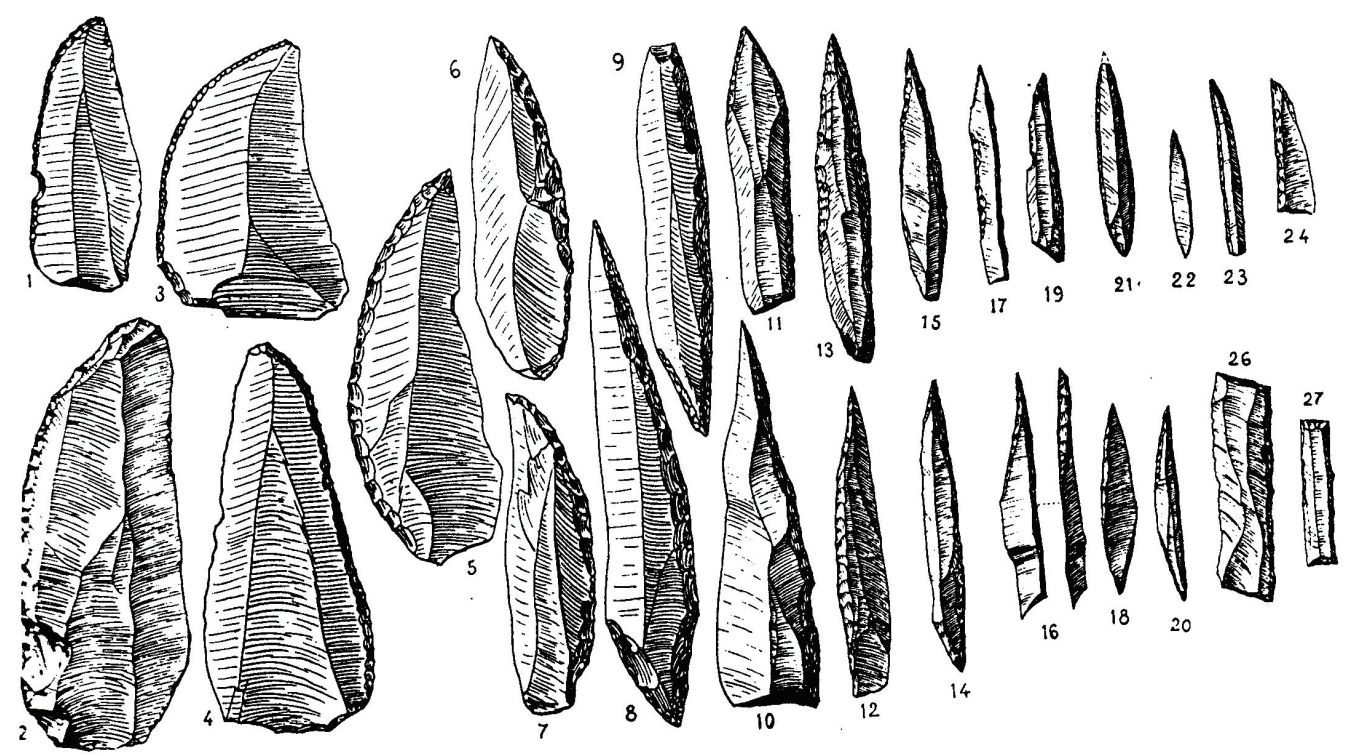

Figura 1.- Ilustración del artículo de H. Breuil (1912), en el que se muestra la evolución morfológica de las puntas auriñacienses.

Paleolítico Superior, ubicándolo entre el Paleolítico Antiguo y el establecimiento de los primeros Neolíticos (Breuil 1912). Además, lo identificó con la llegada de razas más "elevadas" que las precedentes neandertales y con una colonización de Europa occidental en toda regla (Breuil 1912:174-175).

En esta etapa que denominamos particularista (normativista, historicista-cultural, etc. cf. Johnson 2010) se tendió a parcelar o subdividir en diferentes ramas la historia cultural, se identificaron los conjuntos arqueológicos ya no con edades o etapas, sino con "culturas", "complejos culturales", "tradiciones" o "facies", a los que se asociaban tipos humanos. Esta nueva corriente acarreaba la idea de que las innovaciones culturales tienen un origen único que luego se difunde por migraciones o invasiones y, por tanto, todos estos sucesos se podían rastrear a través de los restos arqueológicos (Vega Toscano 2001). Es en esta corriente donde H. Breuil concreta su definición del Auriñaciense y, tal como se va a explicar, donde nacen los términos de Cultura Perigordiense y Gravetiense en los estudios de Paleolítico.

Unos años después, otro de los principales investigadores franceses de comienzos del siglo XX, D. Peyrony, dedicó varios escritos a exponer una nueva teoría de filiación entre el Auriñaciense Inferior (de la Punta de Chatelperron) y Superior (de la Punta de La Gravette) (Peyrony,1933 1936), fruto de sus trabajos en varias cuevas del Perigord (La Ferrassie y Laugerie-Haute). En el transcurso de sus excavaciones, Peyrony intuyó que las industrias de la Cultura de Chatelperron y de La Gravette eran relacionables, por lo que propuso una interpretación en donde las evidencias de la segunda derivaban de la primera, apoyando su argumento estrictamente en la tipología y en una facies documentada en Laugerie-Haute. Estas industrias - caracterizadas básicamente por el retoque abrupto - estaban plenamente representadas en el Perigord, por lo que consideró lógico denominarlas "perigordienses". Llegó a distinguir hasta cinco facies dentro del Perigordiense asignando a la última $\left(\mathrm{n}^{\mathrm{o}} \mathrm{V}\right)$ otras tres variedades $(\mathrm{a}, \mathrm{b}$ y c), según los trabajos llevados a cabo en La Ferrassie. Posteriormente, este esquema se fue complicando aún más, si cabe, al señalar dos ramas diferenciadas dentro del Perigordiense (Peyrony 1946) (Fig. 2).

En su modelo teórico, Perigordiense y Auriñaciense - este último periodo reducido al antiguo Auriñaciense Medio de Breuil- eran culturas sincrónicas y para cada una de ellas se podían distinguir diferentes variantes tipológicas a partir de fósiles-directores óseos (en el caso Auriñaciense) y líticos (en el caso Perigordiense). Asimismo, Peyrony identificó estas culturas con razas humanas, por lo que, desde su perspectiva, el Perigordiense se podía asociar a la raza de Combe-Capelle y el Auriñaciense con la de Cro-Magnon (1936). 


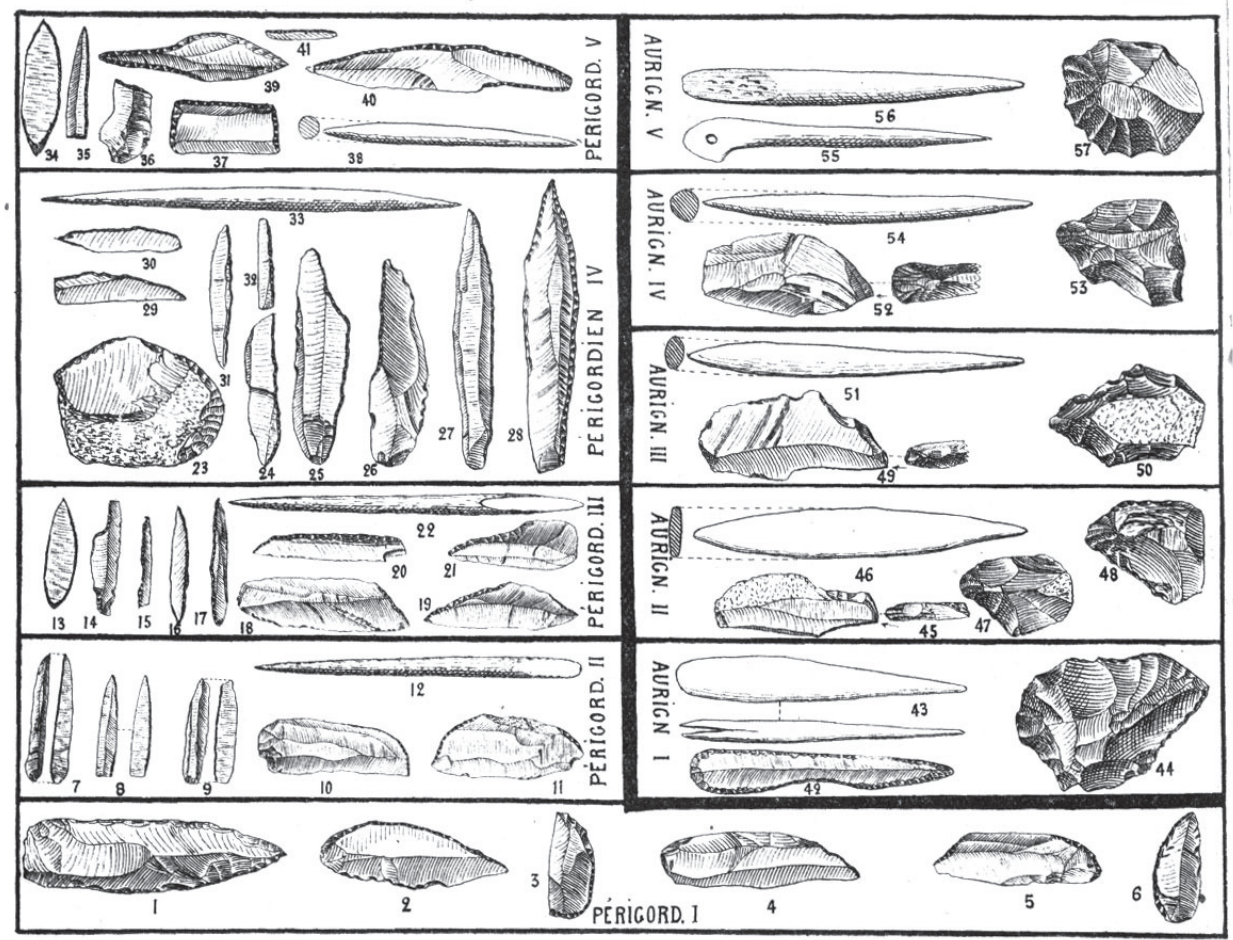

Figura 2.- Modelo de phylum paralelos (Perigordiense y Auriñaciense) diseñado por D. Peyrony (1936).

\subsection{D.A.E. Garrod: la creación de la cultura Gravetiense}

Aparte de la teoría regional francesa, por aquellos mismos años la prehistoriadora inglesa D.A.E. Garrod, discípula de H. Breuil, definió un nuevo modelo de evolución de las culturas del Paleolítico Superior inicial —o antiguo Auriñaciense - que también ha tenido una enorme trascendencia (Garrod 1936).

Garrod señaló su desacuerdo con respecto al esquema de D. Peyrony y empezó a dudar de que la clave del desarrollo de las industrias laminares del Paleolítico Superior europeo se encontrase en el Suroeste francés. Por otra parte, defendió que entre los tres tipos de Auriñaciense de Breuil se constataba una sucesión estratigráfica, por lo que estableció un modelo tripartito de evolución en el inicio del Paleolítico Superior, que quedaría entonces caracterizado por tres tipos diferentes de industrias sucesivas, a saber: Chatelperroniense, Auriñaciense y Gravetiense, a los que consideraba, a su vez, culturas. Además, Garrod (1936) explicó su concepción de las industrias con puntas de La Gravette y Font Robert, a las que consideró extendidas por buena parte de Europa central y oriental e identificaba con el fenómeno de las estatuillas femeninas. En concreto, diferenció dos fases dentro de lo que definió como Gravetiense: una con la conocida Punta de La Gravette y otra, posterior, caracterizada por las puntas pedunculadas (Font-Robert), las puntas de Muesca de Willendorf y las figurillas femeninas denominadas "venus". En el párrafo siguiente se reflejan muy bien estas ideas y su concepción de difusión por toda Eurasia:

Finally, the Gravette-Font-Robert industry has a very wide distribution in Central and Eastern Europe, and its remarkable development in this region points rather to Euroasiatic origin (...) The theory of an eastern centre of dispersion for the Gravettian is based, of course, on this exceptional development in Central and Eastern Europe. I am influenced also by the fact that the female statuettes, the close connection of which with the Upper Gravettian is incontestable, are very abundant in Russia, but occur only sporadically in Western Europe, where they have an unmistakably alien appearance... (Garrod 1936: 829)

D.A.E. Garrod estableció para el Gravetiense una definición, un origen y una de las principales bases de su identificación a lo largo de Europa: el fenómeno de las venus. En resumen, fue la creadora del concepto de unidad e identidad cultural grave- 
tiense. Su propuesta trataba al Chatelperroniense, Auriñaciense y Gravetiense como grandes culturas paneuropeas y pretendía definir centros de origen y difusiones. Por el contrario, el modelo de Peyrony era, en principio, estrictamente regional dentro del Suroeste francés. Esta vocación más amplia a nivel geográfico es una de las principales características que distinguía al modelo de Garrod del de Peyrony, según se recordó en el trabajo homenaje a esta insigne prehistoriadora (Davies 1999). Del mismo modo, no es casualidad que fuera una discípula de Breuil la que promoviera estas ideas, pues ya en el trabajo Les subdivisions du Paléolithique Supérieur et leur signification se defendían este tipo de propuestas de movimientos de pueblos o etnias a una escala que superaba ampliamente el marco francés. En otras palabras, ambos poseían una vocación geográficamente más amplia de miras dentro de los discursos de Prehistoria y un cuidado especial en la estratigrafía y no tanto en la tipología, lo que a la postre se demostraría mucho más acertado.

\subsection{La cuestión perigordiense}

A partir de la segunda mitad del siglo XX los estudios de Paleolítico Superior se realizaron desde una nueva perspectiva: la tipología-estadística. Este nuevo sistema de investigación fue aplicado en primer lugar a los conjuntos musterienses franceses (Bordes 1961), cuyas principales aportaciones ya han sido resumidas en otros estudios historiográficos (Vega Toscano 1988, 2001). Esta nueva corriente implantó los estudios pluridisciplinares, la contextualización paleoclimática de los estratos en el sentido de marco referencial comparativo entre secuencias y la consideración de la industria lítica en el de unidad de estudio inteligible a partir de las frecuencias estadísticas de tipos, es decir, unidades de estudio en sí mismas.

Por lo que respecta al Perigordiense, D. Sonneville-Bordes retomó su estudio en la zona clásica del Perigord bajo este nuevo esquema (Sonneville-Bordes y Perrot 1953, 1954, 1955, 1956; SonnevilleBordes 1960, 1966). El sistema tipológico estadístico confirmó, desde una base aparentemente más sólida, la coherencia de la definición tipológica de las facies del Perigordiense de D. Peyrony.

Unos años después, F. Bordes resumió, desde esta perspectiva, la denominada "cuestión perigordiense" (Bordes 1968). En un artículo exclusivamente dedicado a ello defendió extensamente el modelo de paralelismo entre Perigordiense y Auriñaciense y la visión de tradiciones culturales de largo alcance instaurada por Peyrony. En el inicio de este periodo la hipótesis de coetaneidad Auriñaciense-Perigor- diense quedaba demostrada por la interestratificación entre Perigordiense I y Auriñaciense - a través de las excavaciones de Le Piage y Roc de Combe(Champagne y Espitalie 1967), mientras que en lo que concernía al Perigordiense Superior, F. Bordes sostuvo la sincronía entre el Perigordiense IV y el Auriñaciense III y IV, en función de la correlación de las secuencias de La Ferrassie y de Roc de Combe Capelle, a partir del marco climatoestratigráfico ofrecido por el estudio sedimentológico de los estratos (Bordes 1968). ${ }^{1}$

Al mismo tiempo, F. Bordes defendió la filiación entre el Perigordiense Inferior y el Superior, por la ausencia de evidencias en Europa central y oriental que pudieran ser el origen del segundo y porque, a su juicio, existían numerosos argumentos de orden tipológico que relacionaban a ambos: la similitud morfológica (simetría) entre algunas Puntas de Chatelperron que las vinculaba a Las Puntas de $L a$ Gravette, los mismos métodos de talla laminar, el trabajo del asta por medio de buriles, etc. En definitiva, en aquel artículo, F. Bordes propuso - exclusivamente dentro del contexto francés - la coetaneidad de dos "tradiciones culturales" que habrían evolucionado sin influirse la una a la otra. El Perigordiense era la tradición autóctona, mayoritaria y dominante, mientras que el Auriñaciense era un "fenómeno intrusivo" oriundo de una oleada migratoria externa (Bordes 1968:67-68) (Fig. 3).

En esas mismas fechas se empezaron a realizar excavaciones que supusieron a la postre un importante escollo para la teoría del paralelismo de Auriñaciense y Perigordiense, al establecer un hiato cada vez mayor entre el Perigordiense Inferior (I) y el Superior (IV-V). En este sentido se deben señalar, por ejemplo, las excavaciones del propio matrimonio Bordes en Laugerie-Haute (Bordes 1958) que acabaron estableciendo la posición cronoestratigráfica del Perigordiense Medio (III) como más tardía -adquiriendo por ello en la nomenclatura tipológica el $n^{\circ}$ VI - o las de H. L. Movius Jr. en el Abri Pataud (Bricker 1995), en donde se descubrió un nivel idéntico en este abrigo por encima de la Capa IV (adscrito al Perigordiense Vc). Además, durante los años 50 la misma D. Sonneville-Bordes estableció - en base a su protocolo de análisis - el carácter auriñaciense del Perigordiense II (Sonneville-Bordes 1955), lo cual aumentaba, si cabe aún más, el vacío cronológico entre Perigordiense inferior (I) y Perigordiense Superior (IV).

Por tanto, la ordenación e interpretación que se argumentaba era la originaria formulada en los años 30 por D. Peyrony, incluso cuando el nuevo método de análisis de las industrias y los estudios estratigráficos aportaron grandes anomalías empíricas al mismo, siendo la más flagrante la desaparición del 
Perigordiense Medio (III), fundamental en la hipótesis original perigordiense. No deja de ser paradójico que los partidarios acérrimos de la civilización perigordiense fueran, al mismo tiempo, los que eliminaron las facies intermedias (II y III) de Peyrony.

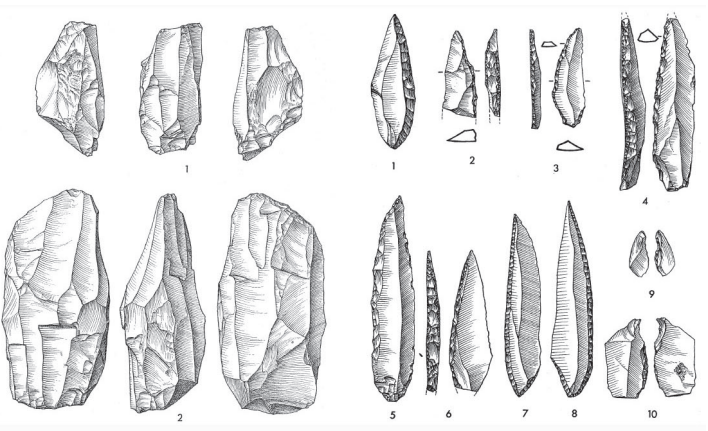

\begin{tabular}{|c|c|c|}
\hline DORDOGNE & LOT & CENTRE \\
\hline A.Y Laugerie-Haute & $"$ & \multirow[b]{4}{*}{ P.Y } \\
\hline \multicolumn{2}{|l|}{ P. YII } & \\
\hline P. VI & P.YI R.de C. c.1 & \\
\hline P. Y & $\begin{array}{ll}\text { P.Y } & \text { R.de C. c. } 2,3\end{array}$ & \\
\hline A.IY & Gravettes (P.IY) R.deC. c.4 & P. "IY" \\
\hline A.III & A. évolué R. de C. c.5 & \\
\hline A. II & A. II $\quad$ R. de C. c.6 & \\
\hline A. I & A. I R. de C. c.7 & Les Cottés \\
\hline A. ${ }^{\circ} \mathrm{O}^{\circ}$ (Ferr. E') & R.de C. c.9-Piage & \multirow[t]{2}{*}{ ? } \\
\hline P.A.1 (Ferr.) & $\begin{array}{ll}\text { R. de } \text { C. } \quad c .10 \\
\end{array}$ & \\
\hline Moustérie & de tradition acheulée & \\
\hline
\end{tabular}

Figura 3.- Arriba, ilustraciones de industria lítica con las que F. Bordes argumentaba —a partir de la tipología - la filiación del Perigordiense Inferior y Superior. A la izquierda se muestran núcleos de Roc de Combe con dos planos de fractura opuestos entre sí (1, Perigordiense Inferior y 2, Perigordiense Superior); a la derecha, similitud morfológica de varios cuchillos de Chatelperron con las Puntas de La Gravette. Abajo: esquema con el que se explicaba la irrupción del Auriñaciense (y sus variantes) dentro de la evolución perigordiense en las diferentes regiones francesas (Bordes 1968).

En resumen, aunque el procedimiento de estudio mejoró hacia un refinamiento del trabajo de campo, la creación de marcos paleoclimáticos y una apurada clasificación del objeto principal de estudio para la Prehistoria de entonces (las industrias), en lo que respecta al marco teórico y las interpretaciones aplicadas, el discurso siguió siendo, en esencia, particularista. En este sentido, no hubo ni debate ni discusión en la concepción o el significado de las recurrencias estadísticas industriales, sino que se heredó la base teórica previa. Nos referimos a la identificación cultural de las entidades halladas o diferenciadas, lo que, recuérdese, era el eje vertebrador de las propuestas anteriores. Por ende, no se puede hablar de cambio metodológico (entendiendo "metodología" como un sistema estructurado de razonamiento) con respecto a la etapa previa — como sí que se había producido antes entre el evolucionismo de Mortillet y el particularismo de H. Breuil con la "Batalla del Auriñaciense" de por medio. Ahora el marco teórico que fundamentaba el protocolo de análisis tipológico-estadístico para el Paleolítico Superior era el mismo que en la etapa de estudio precedente y por ello parece coherente concebir a este tipo de aproximaciones como una continuación del Particularismo del primer cuarto del siglo XX.

Hubo investigadores dentro de la tradición francesa y española que discrepaban con esta visión, por ejemplo Jordá (1954), Delporte (1954), Cheynier (1960) o el propio Leroi-Gourhan (1961), sugiriendo la división de Garrod (1936) de Chatelperroniense y Gravetiense como culturas netamente separadas. Entre los principales escollos a la teoría de Peyrony se citaban la ausencia de identificación de las facies de Peyrony (véase el caso levantino ibérico), las débiles bases existentes para sugerir que el Perigordiense inferior/Chatelperroniense proviniese del Este europeo, la dificultad de defender que dos supuestas culturas hubieran evolucionado sin influirse la una a la otra, las similitudes del Chatelperroniense con las industrias previas musterienses - sugiriendo tácitamente una evolución autóctona en el marco francés-, e incluso se indicaba que aquel pudiera estar relacionado con los Neandertales por las excavaciones de Arcy por el hallazgo de dientes de Neandertales asociados al Chatelperroniense-, etc.

\subsection{G. Laplace: el Gravetiense como Complejo}

Es de resaltar en este tema la propuesta teórica y metodológica que G. Laplace aplicó al tema que estamos tratando, con la denominada hipótesis del Sintetotipo Auriñaciense (Laplace 1966). Su método de análisis se basó también en la tipología estadística, pero a partir de una variante distinta y original: la Tipología Analítica, surgida como respuesta a la clasificación descriptiva de la listatipo de D. Sonneville-Bordes y J. Perrot (Laplace 1956, 1966, 1972). Laplace partía de una premisa idéntica a la propuesta aludida, es decir, la concepción de la industria lítica como objeto de estudio 
inteligible desde la estadística. Para él, mediante una clasificación más objetiva y puramente descriptiva se descubriría la idiosincrasia y leyes de evolución de las industrias, como si de un ser vivo se tratara. Otra aportación de esta nueva formulación analítica es que trató de ser más exacta con respecto al material a analizar y a la terminología utilizada. De esta manera, Laplace creó el concepto de "Complejo", con el que se refería a un grupo de industrias que presentan un equilibrio en el sentido estadístico, mientras que, a nivel teórico $-\mathrm{y}$ esto sí que era una aportación novedosa-, consistía en el resultado de la interrelación dialéctica del desarrollo tecnológico propio de un grupo y su medio externo, concebida esta relación de forma dinámica (Laplace 1956).

G. Laplace tuvo el afán de superar las dificultades y contradicciones que se constataban en la propuesta de D. Peyrony sobre Auriñaciense y Perigordiense (Laplace 1966: 9). Para tal empresa, formuló una teoría específica: el Sintetotipo Auriñaciense. Dicha propuesta teórica se inspiró en esquemas realizados para la Paleontología, como la teoría de la "Cosmolyse" de A. C. Blanc (Ibid.: 263): a partir de entidades o agrupaciones arcaicas heterogéneas y entremezcladas se desarrollan entidades homogéneas y diferenciadas. El análisis estructural de los "complejos" industriales paleolíticos, a través de la Tipología Analítica, permitiría percibir procesos internos de cambio gradual o abrupto de las industrias. Los complejos se correspondían, según los casos, a etapas de amalgama (y cambio gradual) o a unidades coherentes (y estáticas).

La interpretación que obtuvo, en función de la aplicación de su original método, fue la propuesta de la génesis evolutiva interna de desarrollo de las industrias al inicio del Paleolítico Superior, a través de un proceso que denominó como de "leptolitización", en el que el desarrollo de la talla laminar habría sido el principal motor evolutivo (Ibid.: 265). En concreto, desde un estadio denominado como Polymorphisme de base de las industrias de Paleolítico Medio se sucedió una evolución -como la conjeturada por la Cosmolyse- a través de diferentes fases: Phase Préapogéique d'inmobilité relative, Phase Préapogéique d'acceleration brusque, etc., que culminó con los estadios homogéneos ulteriores del Paleolítico Superior, entre ellos, el Gravetiense. En resumen, en una fase definida como Postapogéique de ségrégation et de spécialisation se habría individualizado el Complejo Gravetiense occidental como entidad industrial unitaria (Fig. 4).

En definitiva, la propuesta laplaciana reintroducía una concepción evolucionista por la influencia

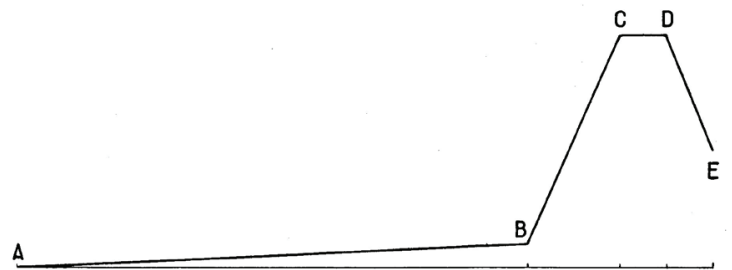

\section{Echelle des temps}

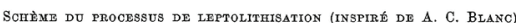

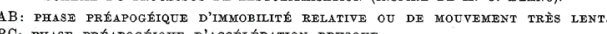

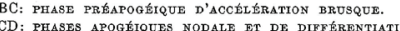

DE: PEASE POSTAPOGEIQUT DE SÉGREGGATION ET DE BPÉCILIISATION.

Figura 4.- Esquema gráfico de las fases definidas dentro de la Teoría del Sintetotipo Auriñaciense según la propuesta de la Cosmolyse de A. C. Blanc (Laplace 1966).

de la teoría mencionada. Desde este punto de vista sí que constituyó un cambio metodológico con respecto al particularismo dominante, pero en lo que se refiere a la comprensión de este proceso evolutivo no aportaba explicaciones, puesto que la propia descripción —el análisis estructural- consistía en su argumentación y la asociación descripción-explicación de evolución de los complejos no tiene una relación de inferencia causal. En otras palabras, una apurada descripción tipológica, por muy elaborada y detallada que sea, no aporta las causas ni motivos de lo que se pretende conocer. Recuérdese que la evolución interna de los complejos se entendía como el resultado de las fuerzas de la inercia social y los cambios del medio (Laplace 1956) pero en la aplicación de su metodología no se apelaba a estos dos factores para explicar las razones de la evolución de los mismos - que hubiera sido lo coherente y novedoso en función de su propuesta teóricasino que se aludía, únicamente, a la propia descripción industrial a través de su refinada tipología que - reincidimos en este aspecto- por sí misma no otorgaba explicaciones. Por tanto, la explicación del cambio recaía en el principio evolutivo mencionado y, por ende, esta visión se puede considerar en cierto modo teleológica, como si la evolución de las industrias al inicio del Paleolítico Superior estuviera ya predeterminada.

Conviene añadir que, paradójicamente, en el caso que hemos ilustrado del Gravetiense en Europa occidental, la teoría del Sintetotipo coincidía en la definición de entidad unitaria gravetiense, aunque lo concibiera como una realidad completamente diferente a la de "cultura" y además otorgara una explicación nueva sobre su gestación. La principal diferencia de G. Laplace con respecto a las propuestas previas - aparte del método de análisis y descripción de las industrias - fue que trató de explicar a partir de una teoría interna original el origen 
mismo del Paleolítico Superior. La argumentación hasta entonces había sido, en exclusiva, la migracionista o invasionista. El propio G. Laplace señaló tempranamente esta situación (Laplace 1956:281).

\subsection{Algunas alternativas procesuales}

Durante los años 70 se formularon algunas variaciones teóricas y acotaciones al modelo instaurado por la tipología-estadística. Sin embargo, el caso de las facies perigordienses contrasta con la interpretación de las facies musterienses, donde se evaluaron diferentes hipótesis aparte de la propuesta de las "tradiciones culturales" (evolución cronológica, adaptación al medio ambiente, variabilidad estacional, etc.) (Bordes 1953, 1961; Bordes y SonnevilleBordes 1970) dando lugar a uno de los debates más controvertidos en Prehistoria durante la segunda mitad del siglo XX (Binford 1973; Bordes 1973; Mellars 1973).

La cuestión perigordiense no generó una discusión de tan hondo calado y las pocas propuestas que discreparon con la visión culturalista dominante no llegaron a cuestionarla del todo. El debate se centró en admitir o no al Perigordiense y Auriñaciense como dos phylum paralelos o en reivindicar las tres culturas de D.A.E. Garrod. El único modelo procesual generado sobre esta problemática fue la propuesta del Noaillense de N. David. Este investigador, que realizó su tesis doctoral sobre la industria lítica del nivel IV del Abri Pataud, concluyó, tras el análisis por el método tipológico-estadístico de las industrias, que dicho conjunto podía interpretarse como la manifestación de una etnia/cultura completamente diferente a la de Perigordiense Superior: la Noaillense (David, 1973 1985; David y Bricker 1987) (Fig. 5).

El modelo de David no fue novedoso por su metodología de análisis industrial - ya que se basaba también en el análisis tipológico-estadísticoni tampoco en su interpretación - en cierto modo llevaba hasta sus últimas consecuencias el modelo cultural de D. Sonneville-Bordes. Fue innovador en el sentido de realizar analogías etnográficas con pueblos indígenas contemporáneos de forma completamente manifiesta (David 1973) ${ }^{2}$. Bien es cierto que esta práctica, en cierta medida neoevolucionista, tampoco era completamente original de la arqueología procesual: los pioneros de la Prehistoria ya empezaron a realizar analogías etnográficas. El mérito de esta nueva propuesta es que con este tipo de analogía introducía cuestiones nunca antes planteadas en la explicación del Perigordiense.

En concreto, David escogió para plantear su modelo noaillense a poblaciones esquimales ca-

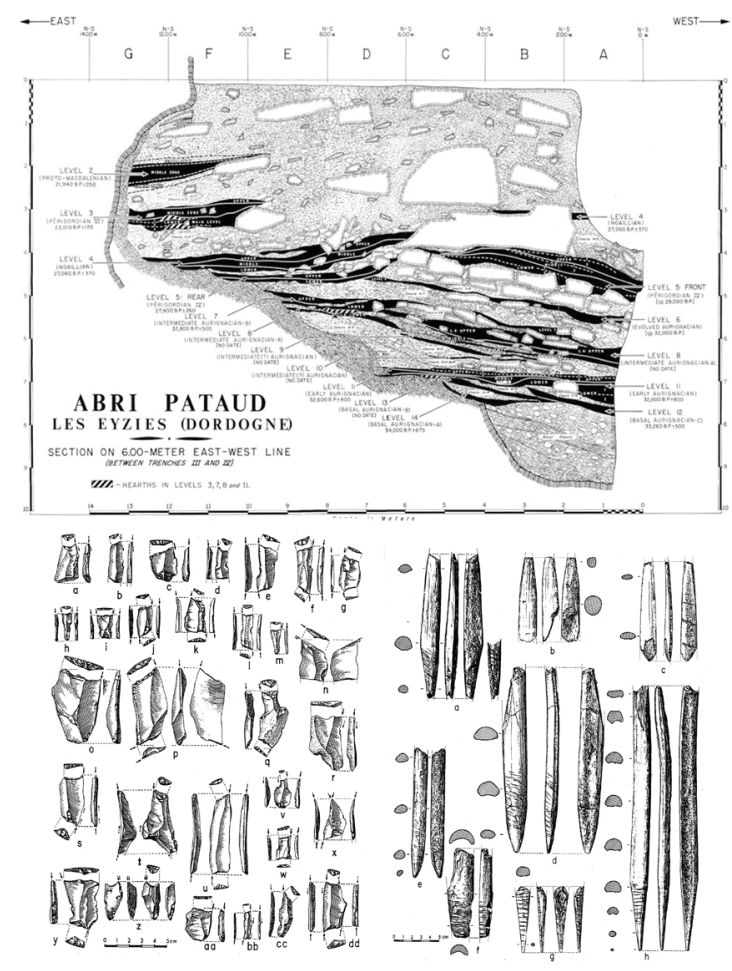

Figura 5.- Arriba, estratigrafía del Abri Pataud. La excavación del equipo americano dirigido por H. L. Movius Jr. vino a confirmar el hiato perigordiense, tras descubrirse un nivel correspondiente al Perigordiense III por encima de la Capa IV (relacionada con el Perigordiense Vc). Es una de las secuencias estratigráficas más largas del Paleolítico Superior inicial del Suroeste francés y fue fundamental en la discusión perigordiense. Abajo, industria lítica (buriles de Noailles) y ósea (azagayas de Isturitz) de la Capa IV del mismo yacimiento, atribuidas por N. David a la Cultura Noaillense (según Movius 1977 y Bricker 1995).

zadoras de caribú. Su razonamiento se basaba en la economía propuesta para los grupos noaillenses que, según estudios de fauna del momento, se habían sustentado en la caza del reno, un animal gregario y estacional equiparable al caribú norteamericano. A partir de estudios etnográficos de los Nunamiut, Nabesna, Athapaskanos y Naskapi propuso una organización de la que denominó "Cultura Noaillense" en bandas con diferentes tipos de sitios complementarios entre sí. Asimismo, a las tres grandes regiones de Europa occidental en las que se había identificado esta facies tipológica (Perigord, Sureste de Francia y Pirineos) las correlacionó con tres grandes tribus que se habrían dividido en pequeños grupos sociales o bandas en determinadas épocas del año.

Aunque la tesis de David fue una elaborada argumentación sobre la diferencia cultural del Noaillense 
y el resto del Perigordiense - una línea largamente tratada por la investigación de los años 50-60, como se acaba de ver-, en su propuesta posterior a partir de la analogía etnográfica incluía aspectos demográficos, económicos, sociales, de ordenación del territorio, etc., nunca antes planteados en las explicaciones para el Perigordiense y, prácticamente, para el resto del Paleolítico Superior.

Además, con esta propuesta teórica como pretexto, introducía también una idea nueva para los cambios en paleolítico, diferente a los modelos migracionistas y evolucionistas previos. La razón para el cambio la entendió como un desequilibrio interno dentro del sistema cultural, fruto de la dinámica interna de un grupo étnico dado, que se generaba a partir de fenómenos históricos específicos o por la acumulación de una tendencia. La fluctuación natural de las poblaciones de presas cazadas —en el caso del Noaillense, las manadas de renos-y de los predadores noaillenses, eran la base de su teoría. Estas fluctuaciones en determinados intervalos pudieron ser de tal grado como para llegar a romper la dinámica de una sociedad de cazadores, causando el colapso de su equilibrio social y por tanto la transmisión cultural de generación en generación, abriendo así la posibilidad de introducción de cambios y la creación de nuevos sistemas culturales sin la necesidad de apelar a difusiones o transmisiones foráneas. En último término su idea era determinista ambiental y por supuesto sistémica, por lo que desde el punto de vista del cambio esta explicación era algo novedoso.

Por otra parte, se debe destacar un artículo - también de estos mismos años - sobre las facies del Perigordiense V de Peyrony (Va: Font Robert, Vb: truncaduras y Vc: Noailles), que vino a añadir una anomalía más al modelo de mitad de siglo XX para el Suroeste francés. En él H. Laville y J.-Ph. Rigaud llamaron la atención sobre el hecho de que las facies del Perigordiense V no se suceden, tal y como propuso Peyrony, sino que en función del estudio estratigráfico de varios yacimientos aquitanos parecían coetáneas, pudiendo tener un carácter funcional y no cultural (Laville y Rigaud 1973). Para Laville y Rigaud la propuesta de Peyrony se trataba de una generalización de la evidencia de La Ferrassie, pero no se debía tomar como una evolución válida única pues los sitios de Le Vachons, Roc de Combe, Battuts y Le Flageolet I combinaban aleatoriamente las facies del Perigordiense V. En definitiva, aquel no representaba una evolución unilineal y sus variantes (las facies) probablemente se debieran a diferentes actividades ejercidas por las "poblaciones" perigordienses. El sistema de correlación entre niveles, al que recurrieron para su argumentación, fue el paleoclimático. A través de la sedimentología $\mathrm{H}$. Laville distinguió diferentes episodios para la pulsación interestadial de
Tursac, pudiendo situar de una manera afinada cada uno de los niveles en cuestión y demostrar que las variedades del Perigordiense V no se sucedían, sino que, en muchos casos, eran coetáneas.

Aunque la denominada cuestión perigordiense ocupó buena parte de la segunda mitad del siglo XX dentro de los estudios franceses del Paleolítico Superior, las interpretaciones no cambiaron sustancialmente durante este periodo, más allá de la sustitución de la categoría cultura por la de tradiciones culturales que hemos señalado. El debate se centró, sin lugar a dudas, en la correcta ordenación diacrónica de las tradiciones auriñaciense y perigordiense. Su interpretación en clave cultural raramente se cuestionó y la introducción de nuevas preocupaciones se limitó a la propuesta procesual de N. David. La interpretación de Laville y Rigaud para el Perigordiense V, que suponía un auténtico escollo a la sucesión de las facies de Peyrony desde la misma metodología paleoclimática bordesiana, pasó desapercibida.

\subsection{La escuela tecnológica}

A partir de los años 70 y 80 se percibe dentro de los trabajos de investigación franceses un cambio en el tipo de preocupaciones de los estudios de Prehistoria, en concreto el interés por "el tiempo breve de lo cotidiano", de los modos de vida, etc. (Cahen et al. 1981; Karlin et al. 1991). Es decir, ya no sólo es importante reconstruir la evolución en el tiempo de las "culturas prehistóricas", sino también su desarrollo sincrónico y "lo cotidiano" dentro de lo cual se incluían las prácticas técnicas.

Quizás el máximo exponente de esta nueva visión son los trabajos desarrollados en los yacimientos magdalenienses de la cuenca parisina (Pincevent, Etiolles, Verberie, etc.) fruto de la escuela paleoetnológica iniciada por A. Leroi-Gourhan. Este etnólogo y prehistoriador no se preocupó, en el ejercicio de la investigación en Arqueología prehistórica, por la definición específica de determinadas culturas o etnias, ni tampoco de su evolución diacrónica (Demoule 2004). Al concebir a la Prehistoria como etnología se alejaba de la preocupación cultural, por la imposibilidad de aprehender dicha entidad conceptual. Desde esa perspectiva era mucho más interesante caracterizar momentos precisos, focalizando la atención sobre sitios y aspectos concretos (el yacimiento, el suelo de habitación,...) (Leroi-Gourhan y Brezillon 1972; Leroi-Gourhan, 1976), o preocuparse por la evolución de determinados caracteres, p.ej las técnicas, como reflejo del comportamiento cultural de las etnias pasadas (Leroi-Gourhan 1989a, 1989b).

La principal aportación de esta corriente es que dentro de los restos arqueológicos la tecnología cons- 
tituye una manifestación privilegiada de las elecciones culturales (Lemonnier 1991; Demoule 2004). Bajo esta perspectiva, los artefactos líticos ya no se consideraron como más o menos característicos dentro de una cultura, sino como reflejo del comportamiento humano en su dimensión técnica, económica y social (Pelegrin 1990).

Algunos de los ejemplos más tempranos de la aplicación de esta metodología son la tesis de J. Pelegrin sobre el Chatelperroniense (Pelegrin 1995) o la definición del concepto Levallois (Boëda 1989). Asimismo, esta aproximación ha introducido nuevos aspectos en los modelos europeos del Paleolítico Superior - aunque todavía de forma muy tímidacomo por ejemplo los económicos y de organización espacial-geográfica (Pigeot 1987; Perlès 1992; Pigeot 2004), casi siempre referidos a la industria lítica.

A partir de esta metodología se han caracterizado y reinterpretado las antiguas "tradiciones culturales" del Paleolítico y, recientemente, las definidas antes como perigordienses/gravetienses, en las obras de Nespoulet (1995), Zilhão (1997), Aubry et al. (1998), Almeida (2000), Klaric (2003), Guillermin (2004), Pottier (2005), Simonet (2009a), Pesesse (2010), etc.

No obstante, muchos de estos trabajos han sancionado, desde la tecnología, la división ya proporcionada desde la tipología-estadística. En cuanto a las hipótesis sobre el cambio - que pueden considerarse como una vía de reflejo teórico-, los estudios de tecnología lítica no parecen haber ofrecido, hasta el momento, propuestas originales con respecto a la trayectoria historiográfica previa, pese a que sus bases teóricas se sustentan en nuevos aspectos.

La tecnología se considera como reflejo de lo social, lo que constituye un giro teórico decisivo, un punto de partida desde el que se pueden trazar otro tipo de explicaciones. Implica dotar de un significado sociocultural a los objetos arqueológicos, lo que supone que su comprensión no se limita a la esfera de clasificación arqueológica y se pueden realizar otro tipo de inferencias. En las anteriores propuestas se consideró como unidad de estudio inteligible a las industrias, pero sin dotarlas de ningún tipo de significado, más que el implícitamente cultural, lo que a la hora de interpretar supuso una vía única de interpretación. Sin embargo, todavía no se ha dado el salto a indagar y otorgar explicaciones a partir de la esfera económica, sociológica, etc., de la que se supone es reflejo la tecnología. De hecho, el esquema teórico al que se suele recurrir es el del particularismo, porque la mayor parte de los estudios de tecnología se siguen ciñendo únicamente a una esfera descriptiva, aunque bien es cierto que tienen en cuenta nuevos elementos. Por otra parte, se concibe a la definición tecnológica como un fin en sí mismo, como si la descripción otorgara una explicación a nivel antropológico. Así, dentro de lo que Johnson (2010) ha denominado Low, Middle y High level theories, las propuestas en tecnología suelen quedar dentro de las Low y Middle level theories (Tostevin e.p.), al practicarse un fuerte descriptivismo y acudir a la arqueología experimental o, raramente, a las equiparaciones etnográficas. Generalmente no se alcanzan otras esferas de comprensión u otro tipo de incógnitas. Sin embargo, para la antropología de las técnicas esta perspectiva ha dado excelentes resultados, dado que se es plenamente consciente de que el discurso no se puede quedar en la mera descripción (Pétrequin y Pétrequin 2002).

En la actualidad, el Gravetiense ha pasado a concebirse ya no como cultura, sino como un macro-tecnocomplejo, fruto de la influencia de esta corriente de estudios. Este tecnocomplejo incluye, a su vez, diferentes fases cronológicas, así como también se empiezan a distinguir diferentes variedades geográficas sincrónicas - como el reciente Rayssiense para los contextos franceses - y se ha comenzado a hablar de "mosaico gravetiense" (Klaric 2003; Klaric et al. 2009). Parece que desde la tecnología la trayectoria es muy similar a la realizada previamente por la tipología. Esto no constituye un problema en sí, pues parece lógico que cada zona geográfica en el espacio y en el tiempo diera lugar a unas características técnicas particulares $y$, por ende, a unos caracteres socioeconómicos específicos; es más que pertinente que esta variabilidad sea definida y refinada. Sin embargo, lo que parece preocupante es, por un lado, que no se intente llevar más allá las implicaciones de las descripciones tecnológicas y, por otro, que no se discutan las bases de la pretendida unidad gravetiense, como sí se ha hecho para otros momentos de la Prehistoria, véase el ejemplo del Mesolítico (Zvelebil 2005). Desde los 80 parece que se ha superado casi definitivamente la antigua noción de civilización perigordiense (y, por tanto de phylum paralelos) y tácitamente la comunidad científica ha re-adoptado el término de "Gravetiense" mientras que, por el contrario, el término "Perigordiense" ha quedado prácticamente en desuso y con carácter obsoleto o regional, ceñido en todo caso a donde se originó: el Perigord. Incluso en el contexto francés, donde la propuesta de D.A.E. Garrod fue arduamente discutida y rechazada de forma virulenta, se ha admitido ampliamente el término de Gravetiense. La nueva metodología de conocimiento debería aportar un discurso novedoso a este respecto, pero la sensación que otorga la actual ordenación es la de una re-elaboración de las antiguas facies perigordienses, antes culturales y ahora cronológicas (véase el caso francés, Fig. 6).

Otra prueba de que la metodología historicista no ha sido totalmente superada por los partidarios de la 


\section{PERIGORDIENSE $>>>>$ GRAVETIENSE}

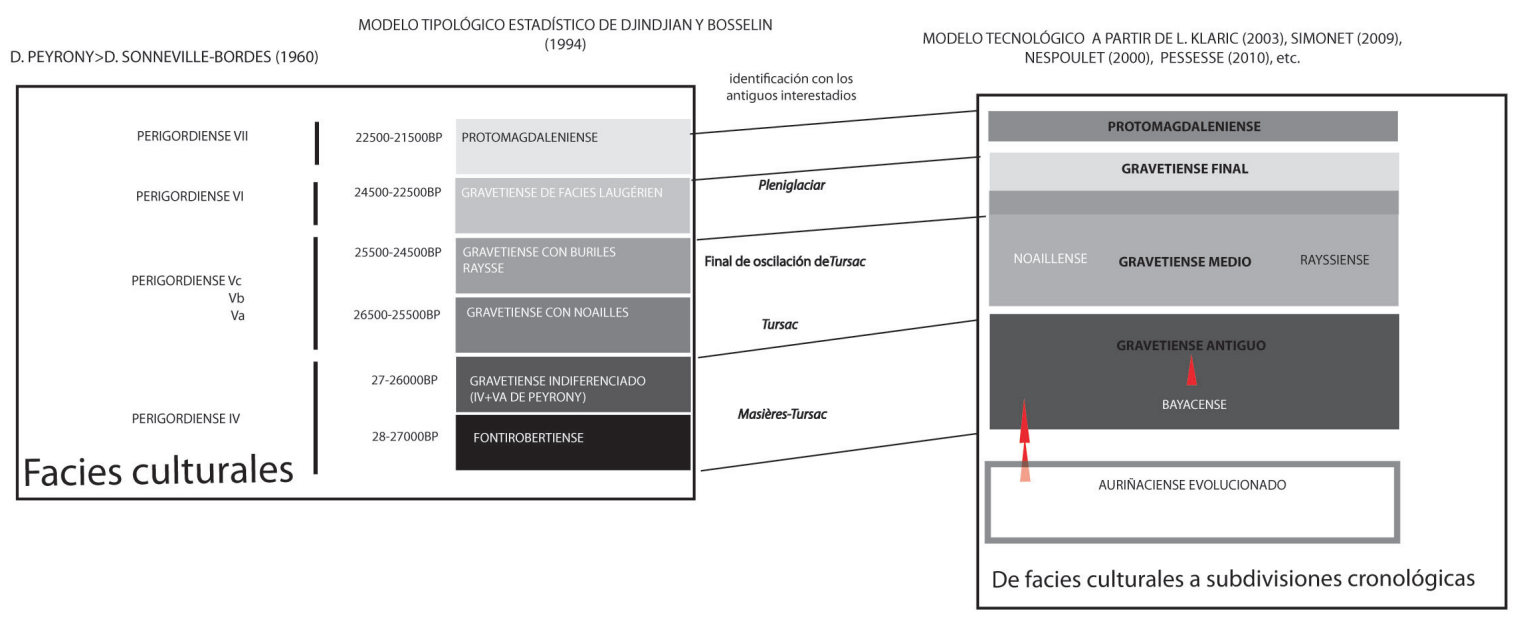

Figura 6.- Evolución de la concepción del Perigordiense $>$ Gravetiense en el marco francés: las facies culturales han sido redefinidas por los modernos estudios de tipología estadística y tecnológicos, sin embargo, las subdivisiones actuales poseen un carácter ambiguo, mezcla de lo cultural y cronológico.

tecnología es que una de las principales preocupaciones sigue siendo la determinación del "origen" primigenio del Gravetiense. Véanse, a modo de ejemplo, las recientes propuestas y discusiones de Pesesse (2010) o Moreau (2010). Sorprende este interés, cuando desde los principios de la Paleoetnología esta temática debería acarrear escaso interés o, más bien, no se debería discutir tanto desde la perspectiva de la tecnología como de la cronoestratigrafía y las técnicas de datación, que son las que están dando, de hecho, argumentaciones contundentes, como por ejemplo la reciente datación de los niveles basales del Abri Pataud (Higham et al. 2011).

\section{Los pilares de la visión unitaria del Gravetiense}

En los años 80 el investigador belga M. Otte adoptó de nuevo la visión paneuropea de la "tradición gravetiense". Esta propuesta quedó perfectamente definida en un artículo de L'Anthropologie (Otte 1985), donde la presentó como un fenómeno homogéneo que afectó a Eurasia por causa de desplazamientos poblacionales o migraciones. Otte planteó que esta identidad se percibe no sólo por la tipotecnología, sino también por las manifestaciones estéticas y simbólicas, tan relevantes y específicas en aquel momento del Paleolítico Superior. En su interpretación concibió a las industrias chatelperronienses y uluzzienses como "antecesoras" del Gravetiense -idea que recuerda a la de D. Peyrony- y distinguió dentro del mismo tres grandes provincias: la zona suroeste france- sa (Perigordiense), la zona central (de Moravia y Austria) y la zona norte europea.

La propuesta de Otte puede entenderse como una vuelta al modelo cultural y particularista de Garrod (1936), no sólo porque retomó la terminología de "Gravetiense" sino porque contempló este fenómeno como una tradición cultural paneuropea que se difundió por migración desde un único origen oriental (Otte y Keeley 1990).

El modelo de M. Otte ha pervivido y está vigente en la actualidad, tanto a partir de las propuestas de este mismo autor como las de J. Kozlowski (2005), que define al Gravetiense como cultura unitaria sucesora del Auriñaciense. Asimismo, A. Simonet ha defendido recientemente esta concepción unitaria a partir del estudio tecnológico del Gravetiense pirenaico (Simonet 2009a, 2009b). Para él, la unidad del Gravetiense se define partiendo de tres aspectos fundamentales: gestión compleja del territorio evidenciada a partir de "hábitats-santuario", deificación de la capacidad generatriz femenina — patente por las venus gravetienses - e importancia de las armas dentro del equipamiento técnico y de la esfera simbólica (Simonet 2009b).

Esta visión de "macrocultura" paneuropea parece sustentada, en buena medida, en la relación que se establece entre los hallazgos e investigaciones de Europa occidental con los de Centroeuropa y de Rusia. La cultura pauloviense morava, la de Mal'ta siberiana o kostikiense rusas (Svoboda 1994; Svoboda et al. 2000; Vasil'ev 2000) quizás sean los ejemplos más emblemáticos con 
los que se han equiparado los restos de Europa occidental.

El Pauloviense moravo tiene una larga tradición de investigación desde los trabajos de K. Absolon en los años 20 del siglo pasado (Oliva 2004). Yacimientos como Dolní Věstonice I y II, Paulov I y II, Milovice I o Předmostí han aportado hallazgos sorprendentes que manifiestan el desarrollo de una compleja sociedad cazadora-recolectora. Entre sus vestigios destacan los hallazgos de viviendas bien estructuradas, hogares, primeros restos textiles y cerámicos, enterramientos múltiples, amplios repertorios de instrumentos líticos, etc. (Svoboda 1994; Soffer 2000; Svoboda et al. 2000; Svoboda 2007). Estos enclaves suelen estar asociados a abundantes restos óseos de mamuts cuya interpretación ha variado desde elementos constructivos de cabañas hasta probables restos de caza relacionados con manifestaciones rituales (Oliva 2000, 2004). Otros elementos característicos de esta presunta cultura son las figurillas femeninas (las venus) y animales, a lo que se añade una extensa panoplia de arte mueble, evocadora de un mundo simbólico y religioso altamente elaborado.

El Pauloviense se desarrolló, según la información de Willendorf II — única secuencia estratificada conocida para esta área- y de otros sitios con un sólo nivel de ocupación, entre el 30-25 ka 14C BP. Luego parece que se desplazó hacia el este, evolucionando en yacimientos con una cultura material diferente y notablemente más temprana: el Willendorfiense-Kostikiense (Svoboda et al. 2000).

La cultura siberiana queda representada por sitios como Mal'ta o Buret', en la cuenca del río Angara. Posee una cronología más reciente (Temprano Sartan, entre el 25-22 $\mathrm{ka} \mathrm{C}^{14} \mathrm{BP}$ ) y se ha subrayado su especificidad en materia de industria lítica - entre las que se encuentran elementos "arcaizantes" como el método Levallois o instrumental sobre lasca- y arte mueble también particular (según los estudios de Abramova, citados en Vasil'ev 2000).

La visión "paneuropea" del Gravetiense ha relacionado estas manifestaciones culturales con la de Europa occidental si bien estas correlaciones suelen basarse en rasgos muy generales. Existen varios factores que parecen tener un peso muy fuerte en esta moderna percepción unitaria del Gravetiense y que han conducido a establecer estos puentes comparativos. A nuestro entender, son tres los aspectos que otorgan esta perspectiva de aparente unidad: los restos de hombres anatómicamente modernos, el arte mueble y parietal y la interpretación de las industrias. Con todo, esta problemática no parece tan sencilla como plantean los partidarios del paneuropeísmo, como veremos al repasar estos tres pilares de manera más detallada:
I. Su asociación con el Homo sapiens es clara, ya que el Gravetiense es la primera etapa del Paleolítico para la que no existen dudas con respecto a su directa asociación con nuestra propia especie, algo que para el Musteriense y para el Auriñaciense no se puede demostrar de forma contundente; incluso los famosos restos del abrigo de Cro-Magnon - por poner el ejemplo más característico- se han demostrado como de "cronología" gravetiense (Henry-Gambier et al. 2006). Este factor puede otorgarle un primer carácter de unidad. Por otra parte, es evidente que el desarrollo, a lo largo del periodo $30-21 \mathrm{ka} \mathrm{C}^{14} \mathrm{BP}$, de diversas manifestaciones funerarias, asociadas a un tratamiento complejo con respecto a los muertos, refuerza aún más si cabe esta idea de unidad. Estas manifestaciones funerarias se entienden como un fenómeno global identificable para Europa occidental y oriental. Así, se comparan sin problemas los enterramientos de Siberia (Sungir, Mal'ta), Chequia (BrnoII, Paulov II), Portugal (Lagar Velho), Italia (Balzi Rossi, Arene Candide...), etc. (Zilhão 2005; HenryGambier 2005), pese a que todos ellos posean cronologías y manifestaciones muy específicas, según ha resaltado la investigación paleoantropológica.

II. Del mismo modo, se debe recordar la importancia del arte en su concepción, algo lógico por suponer, sin duda, una base fundamental en todas las definiciones de cultura, al apelar directamente a la esfera simbólica. Uno de los principales elementos que han contribuido a esa visión de unidad, desde su primera definición, son las conocidas venus gravetienses (Breuil 1912; Garrod 1936; Delporte 1982), pese a que existan áreas europeas para las que no se han documentado hasta el momento, como es la Península Ibérica (Peña 2011). Es a partir de estudios estilísticos o iconográficos de estas estatuillas como se vinculan zonas muy alejadas entre sí en el espacio y en el tiempo. El argumento del estilo lleva a la concepción de esta cultura como una civilización o gran religión. Como ejemplo específico se puede citar el estudio comparativo de las venus de Balzi Rossi que defendió el paralelismo entre Kostienki, Mal'ta o Paulov (Mussi et al. 2000). Esta coherencia estilística también ha servido como argumento para teorías de corte procesual. Las venus gravetienses desde esta perspectiva constituyen el reflejo de la interacción e intercambio de información y de aquí su similitud estilística. Según C. Gamble, serían el resultado de la existencia de redes sociales de largo alcance, un recurso o forma de adaptación a las duras condiciones del Pleniglacial Superior al inicio del Paleolítico Superior (Gamble 1982, 2001).

Sin embargo, estas teorías de unidad estilística a partir de las venus han sido criticadas por investigadores del área oriental europea. Así, O. Soffer ha manifestado su escepticismo a partir de estudios 
estilísticos de detalle de las figurillas (Soffer 1987) sobre la unidad iconográfica de estas obras. De igual forma, ha remarcado como éstas no se circunscriben a una horquilla cronológica acotada, sino que por el contrario su evidencia se puede rastrear a lo largo de más de 10000 años ${ }^{3}$.

Otro de los pilares de esta visión unitaria es el arte parietal. Quizás la manifestación más citada asociada al Gravetiense sean los denominados "Santuarios de manos", como Gargas, Cosquer, Fuentes del Salín, Paglicci, Maltravieso, Fuente del Trucho, etc. (Moure y González 1984-1999; Onoratini y Raux 1992; Ripoll et al. 1999; Palma di Cesnola 2001; Ripoll et al. 2001; Foucher 2005). De nuevo, éstos tampoco son extrapolables a nivel paneuropeo, pues sólo se han documentado en ciertas localizaciones del Midi francés, Perigord y Península Ibérica.

III. Por último, es necesario llamar la atención sobre el elemento considerado como más importante en la articulación de los discursos en Prehistoria hasta el momento: la industria lítica. El Gravetiense/Perigordiense se caracteriza por la proliferación de un tipo de retoque que ha sido considerado como marcador cultural más sobresaliente en la mayoría de todos los estudios realizados: el retoque de dorso. Esta fue la base de la argumentación y de su primera distinción particularista. Hay que recordar, sin embargo, que desde su primera definición el Perigordiense fue una de las culturas del Paleolítico Superior con un mayor número de "facies", es decir, de variantes industriales. Ahora bien, algunas de ellas en la práctica apenas presentan dicho retoque. Por otra parte, tampoco hay que olvidar que se ha señalado en numerosas ocasiones la "regionalización" de esta supuesta cultura a partir precisamente de las industrias. Algunos de los ejemplos ya los hemos citado, como el Pauloviense moravo, el Perigordiense y el Bayacense francés, el Kostikiense ruso o, incluso, nuevas definiciones que se van añadiendo en los últimos años, como el Fontesantense portugués, el Rayssiense francés, el Noaillense (perigordino y vasco-pirenaico), las variantes del Midi, etc. Si atendemos estrictamente a la tipotecnología lítica se debería hablar de un regionalismo extremo y no de una unidad manifiesta.

En definitiva, actualmente se admite la existencia de la unidad del Gravetiense tanto por los partidarios de la tipología-estadística, que ha continuado vigente (vid. entre otros Djindjian y Bosselin 1994; Arrizabalaga 1995; Djindjian 1999; Noiret 2007, Fullola et al. 2007), como por la supuesta nueva tendencia tecnológica (Nespoulet 1995; Zilhão 1997; Almeida 2000; Klaric 2003; Pottier 2005; Simonet 2009a; Moreau 2010; Pesesse 2010). Ambos identifican ahora al Gravetiense con un tecnocomplejo, si bien los fundamentos teóricos en los que se cimenta esta unidad son muy similares a los empleados antaño (Fig. 7). Ambas propuestas están otorgando una subdivisión de este tecnocomplejo unitario y paneuropeo en dos sentidos: diferentes tecnocomplejos menores en su dilatado desarrollo (que son una re-definición, en muchos casos, de las antiguas facies) y diferentes variantes geográficas a lo largo de Eurasia. Además, la industria lítica sigue siendo el principal factor para realizar esta ordenación, pese a que las evidencias gravetienses otorguen un registro material mucho más rico que otros períodos de la Prehistoria.
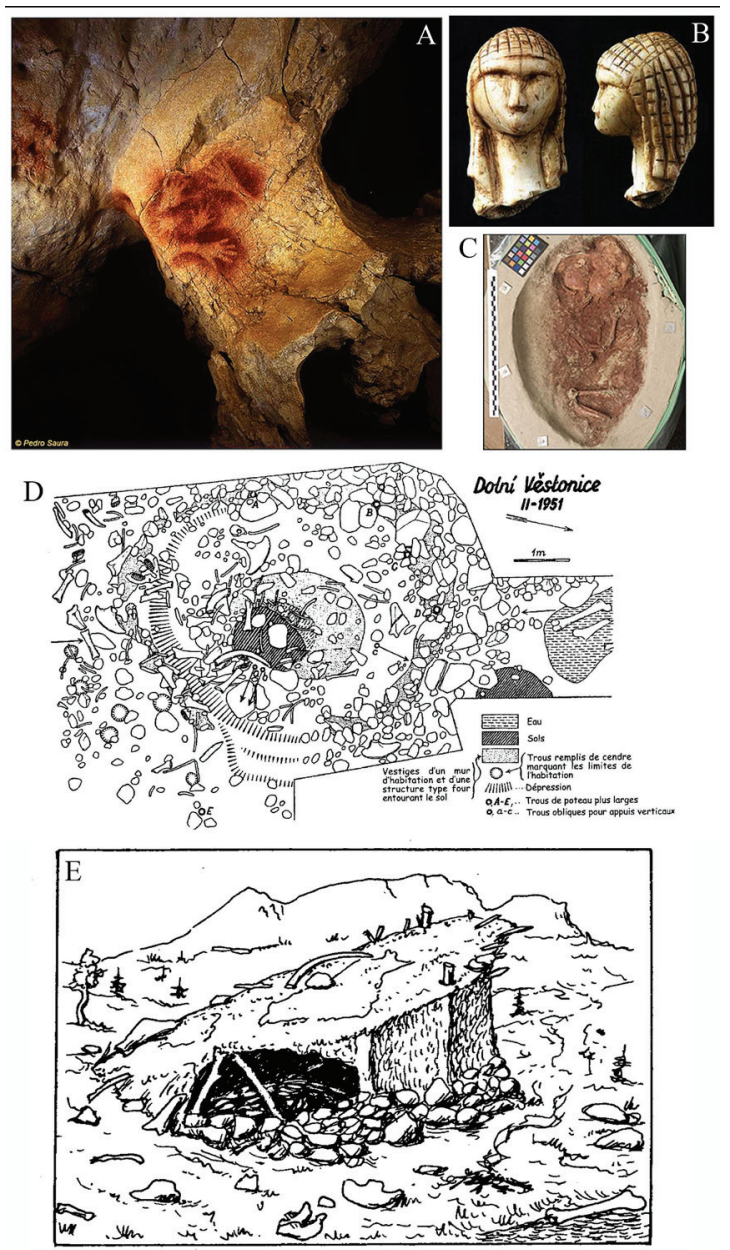

Figura 7.- Distintos elementos relacionados con la macro-cultura Gravetiense. A. Panel con manos en negativo de Fuente del Salín (Fotografía Pedro Saura, tomada de web Lycr). B. Venus de Brassempouy o de la capucha, encontrada en la Cueva de Pape por E. Piette, imagen tomada de Simonet 2009a. C. Enterramientos de neonatos de Krems-Wachtberg (Austria) (Einwögerer et al. 2006). D y E. Estructuras de Dolní Věstonice documentadas por B. Klíma (Leroi-Gourhan, 1976; Oliva, 2004). 


\section{De cultura a tecnocomplejo}

De la síntesis anterior se desprende que parece existir cierta confusión en la terminología empleada en Paleolítico Superior para la denominación y propia concepción de las culturas que lo componen. De hecho, quizás el Gravetiense sea uno de los mejores exponentes de esa confusión. Esta situación pasa desapercibida y es muy raro encontrar artículos o investigaciones que traten de indagar en las bases de la nomenclatura o en los conceptos manejados asumidos de forma general o acrítica, es decir, en las implicaciones que posee utilizar determinada terminología y, en consecuencia, la teoría subyacente a la misma. Considerar esta cuestión solucionada o irrelevante es caer en un error desde el principio, puesto que si no se sabe o define lo que se quiere llegar a saber, difícilmente se podrá identificar, rectificar, matizar o discutir el objeto de estudio que se pretende conocer.

Las definiciones de "cultura" en Prehistoria parecen estar íntimamente relacionadas con las propuestas realizadas a partir de la Antropología. Esta es la percepción que poseen un gran número de especialistas (vid. Watson 1995). A grandes rasgos, se puede decir que se han producido cuatro grandes tendencias en Antropología, reinterpretadas de forma desigual en Prehistoria: las politéticas y, a partir de la segunda mitad del siglo XX, las sistémicas-funcionalistas, las estructuralistas y las hermenéuticosimbólicas. Las que más han influido, en el caso del Paleolítico Superior, han sido, en nuestra opinión, las dos primeras, si bien, su aplicación al ámbito de la Prehistoria no ha sido exactamente en el sentido antropológico en el que se inspiraron, como en ocasiones se ha pretendido.

Dentro de las primeras tendencias cabrían como buenos ejemplos las pioneras de Tylor o de Mauss; para Tylor, cultura significaba "that complex whole which includes knowledge, belief, art, morals, laws, customs, and any other capabilities acquired... as a member of society" (Tylor 1871:1). Mientras que las definiciones sistémicas o funcionalistas en Antropología representadas por Malinowski o Radcliffe-Brown - surgieron años antes que las propuestas de la Nueva Arqueología, que consideró a las culturas de forma sistémica, siendo quizás la definición más citada la de L. White: "culture as man's extrasomatic means of adapatation" (White 1959).

El empleo de este concepto en Paleolítico Superior fue, en un primer momento, el de aquellas primeras definiciones, es decir, cultura como suma de elementos de diferente índole, tanto de entidades materiales como intangibles. Por tanto, es una definición politética, en el sentido de concebir una unidad a partir de la adición de diferentes caracteres comunes (Johnson 2010). Llevado a la práctica arqueológica esa definición de cultura se aplicó a partir del reconocimiento de una suma de elementos característicos exclusivamente materiales, de tipos. De esta manera se eludía la alusión a todos los elementos intangibles que también incluía la definición antropológica. Esta fue una primera fórmula "arqueográfica" de acercamiento a esta problemática, vinculada a las tradiciones de conocimiento que hemos denominado aquí como particularistas.

Asimismo, a principios del siglo XX en los estudios de Paleolítico caló hondo un nuevo término prestado de la Geología y utilizado profusamente, al que también hemos hecho mención: las "facies". Este nuevo concepto se empleó para describir a las variantes líticas del Musteriense y, como se ha explicado, a las del Perigordiense. Este término -que aún hoy suele estar ligado a los razonamientos e interpretaciones a partir de las industrias líticasse traduce en diferentes realidades dentro de los estudios de Paleolítico. De este modo, puede llegar a ser cosas muy diferentes: el estilo de un tipo de talla, la repartición estadística de diferentes tipos de útiles, la presencia anormal de un determinado útil, la elaboración cuidada de aspectos durante la talla, etc. (Lenoir 1974). El concepto "facies" se presentó en los estudios de Paleolítico íntimamente ligado al de "cultura" y en su aplicación se hacía muy difícil distinguir cuáles eran los criterios que llevaban a los investigadores a discernir cuando una variación en el registro alcanzaba el grado de facies y cuando alcanzaba el de cultura. Un buen ejemplo de esta confusión es que determinadas facies tipológicoindustriales han pasado, a lo largo de la historia de la disciplina, a la categoría de culturas o civilizaciones en función de los autores. Un caso evidente es el que hemos expuesto del Noaillense, una facies tipológico-industrial para D. Sonneville-Bordes y D. Peyrony (Perigordiense Vc), que ascendió a la categoría de cultura para N. David, a partir de idéntica metodología (mismo sistema de análisis de atributos líticos).

Según se observa, todos estos conceptos son arbitrariamente empleados y no se suele explicitar a qué se está haciendo referencia. Esta situación se genera, en nuestra opinión, por dos motivos que pasamos a explicar. En primer lugar, existe una dificultad inherente a la propia definición de cultura a la que antes aludíamos. Las definiciones clásicas -que todavía perduran implícitamente en los discursos - hacen referencia a aspectos materiales e intangibles. Poseen un componente ideal - lo que se ha denominado por algunos autores el carácter inconsciente de la cultura (Perrineau 1975)-, mientras que la evidencia arqueológica es una ma- 
nifestación real, tangible. Resulta muy difícil argumentar adecuadamente su existencia si no se sobrepasa el estadio descriptivo al que se suelen ceñir, en exclusiva, los estudios. Es decir, si no se sobrepasa la atención más allá del objeto arqueológico. Para llevar a cabo satisfactoriamente la adopción de esas definiciones antropológicas, en las que se supone que se ha basado la disciplina, se deben establecer nexos teóricos entre el objeto formal de conocimiento y aspectos que interesa llegar a conocer como estructura social, tipo de economía, etc. Pero, generalmente, estos enlaces no se establecen y la asociación entre estos dos órdenes de entidades cognoscibles se lleva a cabo a través de juicios de valor o tan sólo de forma implícita en las argumentaciones.

En segundo lugar, en Paleolítico Superior se suele hacer una "ecuación” errónea: ordenación arqueológica igual a ordenación cultural pasada. En otros términos, el principal problema es que se ha pasado de un ordenamiento o seriación tipológicodescriptiva a la asunción del mismo como realidad en el pasado, lo que no constituye una relación necesaria. El uso de categorías clasificadoras arqueológicas termina identificándose o confundiéndose con la propia interpretación (Vicent 1982). Se manejan las fases tipológicas o tecnológicas como si se tratasen de verdaderas culturas, cuando esta asociación requiere una justificación. El concepto de "facies" ilustra satisfactoriamente toda esta confusión, una categoría arqueológica evidente división material de la industria lítica de un conjunto por diferentes razones (Lenoir 1974) - se convierte automáticamente en realidad cultural o antropológica, es decir, cambia de estatus a través de una analogía sin justificación aparente. Toda esta situación trae consigo un error de mayor alcance: el hecho de confundir categorías tipológicas con culturales acarrea, implícitamente, que la discusión se realice en el estadio descriptivo, en el referente a los puros datos, puesto que, ilusoriamente, parece que en dicho nivel se realiza una discusión teórica. En otras palabras, se contempla a la descripción como un fin, como una propuesta teórica en sí misma. Esta situación es fruto de la confusión entre el objeto formal de estudio de la disciplina y el objeto teorético. Bajo esta forma de actuar se está considerando, aparentemente, en exclusiva al resto arqueológico (objeto formal), lo que deriva en un uso taxonómico del término "cultura". Esta concepción, a su vez, configura a la Prehistoria como disciplina analítica, puesto que sólo se apela a los objetos que contiene el registro empírico (Vicent 1985). Sin embargo, esto no es del todo cierto, ya que sí se suelen realizar teorizaciones y conjeturas más allá del puro registro material. El problema es que aquellos no se justifican ordenadamente a través de un marco teórico especificado o establecido en un estadio previo; así como tampoco se explicita la concepción de cultura que se está tomando, supuestamente, en consideración.

De este modo, resulta imposible refutar o argumentar las bases sobre las que se apoyan los investigadores para realizar sus aserciones. Por esta razón, un conjunto puede ser contemplado por algunos como cultura, por otros como facies, por otros como tradición, por algunos como horizonte o mero conjunto industrial, etc. En resumen, no suele generarse una discusión sobre el significado de las entidades que se están describiendo y, al final, la concepción de las entidades estudiadas queda, de nuevo, a expensas de un juicio de valor.

Recientemente, los antiguos términos de "tradiciones culturales" o "culturas" han sido reemplazados por el de "tecnocomplejos", término creado por Clarke haciendo alusión a un conjunto de culturas que presentan una serie de características comunes (Clarke 1968). Con todo, "tecnocomplejo" es empleado en los estudios de Paleolítico con otro sentido, como sinónimo de tradiciones técnicas de largo alcance, fruto de la relativamente reciente corriente tecnológica. En otros casos, sin embargo, parece estar haciendo alusión a los "complejos" leptolíticos definidos por G. Laplace, a los que se les ha añadido una carga teórica nueva al ponerse el énfasis en la tecnología.

Aunque no sea objeto de frecuente debate, es llamativo constatar que la mayor parte de los tecnocomplejos coinciden remarcablemente bien con las anteriores divisiones culturalistas, pese a restringirse a un aspecto concreto de la antigua definición politética de cultura (la esfera tecnológica) y partir de unas bases teóricas a priori diferentes a las del particularismo. Esta situación parece indicar que se han sustituido las formas descriptivas del material - supuestamente fruto de un giro metodológicopero parece evidente que la carga teórica que llevan aparejado estas nuevas metodologías no se ha llevado hasta sus últimas consecuencias, puesto que las subdivisiones son muy similares a las previas. Todo esto, en términos epistemológicos, no es comprensible, puesto que es imposible que dos metodologías de investigación radicalmente distintas coincidan en sus conclusiones, dado que, a la fuerza, poseerán diferentes problemáticas y diferentes concepciones de certeza, del mismo modo que parece imposible que dos lenguajes diferentes coincidan en sus terminologías.

Quizás el planteamiento más acertado sea abandonar la aproximación holística de cultura —que ha pervivido hasta la actualidad - y centrarnos en 
aspectos concretos de sus manifestaciones. El problema debería residir más bien en definir muy bien lo que se quiere llegar a conocer $\mathrm{y}$, precisamente, el concepto de cultura es tan sumamente amplio y vago que ha generado una enorme confusión en este sentido, cerrando así la entrada a nuevos modelos teóricos. La cuestión no estriba en elegir una buena definición de cultura en el sentido antropológico que se ha pretendido, pues cada vez más se ha contemplado como un imposible incluso para la propia Antropología (Geertz 2005). Tampoco consiste en un apurado sistema de clasificación arqueológico de ordenación, como en ocasiones se ha planteado. La pura descripción no otorga explicación: la taxonomía botánica en Biología no es un tipo de Ecología, sino una forma de clasificación. Lo que es interesante, en contrapartida, son las claves teóricas que rigen esos principios de ordenación, porque establecen tanto lo que se quiere llevar a saber como la forma de analizarlo.

\section{Conclusiones}

Con todo lo visto hasta ahora y en síntesis, se puede afirmar que parece que el esquema que ha quedado en la ordenación del Paleolítico Superior inicial en Europa occidental - al menos, el mayoritariamente aceptado - es una vuelta al modelo tripartito del primer tercio del siglo XX planteado por D.A.E. Garrod pero traducido a la nueva corriente de tecnocomplejos o tradiciones técnicas: de industrias de transición, auriñacienses y gravetienses. También puede decirse que se trata del antiguo modelo de Breuil (1912), como otros autores ya han señalado (Djindjian 2002) —obviando llamativamente la reformulación de Garrod.

En el caso concreto del Gravetiense no deja de ser paradójico que una supuesta "cultura" definida desde el principio con numerosas facies líticas se haya contemplado a lo largo de la historia de la disciplina prehistórica como un fenómeno unitario. Esa idea de unidad o de identidad cultural parece, al menos en parte, una herencia de las primeras interpretaciones sobre este fenómeno. Con este razonamiento no se está negando de partida que pudiese existir una identidad cultural gravetiense o que efectivamente acaeciesen préstamos y oleadas de saberes compartidos en buena parte de Eurasia (Peña 2011), sino que se está afirmando que las unidades de estudio que empleamos son conceptos heredados a partir de la definición de comienzos del siglo XX, a los que se les otorgó, arbitrariamente, el carácter de grandes culturas y que, a nuestro entender, deberían estar en constante revisión (Roebroeks y Corbey 2000).
La realidad actual en los estudios de Paleolítico Superior es que aquella concepción (historicistacultural) no ha cambiado ni ha sido seriamente revisada y, prueba de ello, es que se sigue hablando del "origen" único y primigenio del Gravetiense por muchos investigadores (Otte y Keeley 1990; Djindjian et al. 1999; Kozlowski 2005; Simonet 2009a).

A través del hilo conductor de las industrias el fenómeno del Gravetiense ha sido contemplado como edad, cultura o etnia, tradición cultural $\mathrm{y}$, actualmente, como tecnocomplejo o tradición técnica. Por lo que se ha visto, parece que subyace la concepción de unidad, la identificación con un fenómeno único, pese a que la terminología y la metodología hayan ido cambiando.

Cabe preguntarse si a través de la cultura material esta visión está justificada, lo que debería ir acompañado de un análisis en profundidad de diferentes aspectos dentro de la evidencia arqueológica. La dimensión tecnológica parece una buena alternativa, pero debería incorporarse a otro tipo de información que también ofrecen los yacimientos adscritos al Gravetiense: contextual, paleoecológica, faunística, simbólica, etc., que parecen tener, todavía, un peso menor en las explicaciones. Del mismo modo, la tecnología debería llevarse más allá de la mera descripción, es decir, indagar en las implicaciones que supone la variabilidad técnica definida.

Por otra parte, se debe llamar la atención de que tal y como se define actualmente este fenómeno cultural supera los 10000 años de desarrollo y abarca la extensión de toda la Península europea de Eurasia, incluida la actual Rusia y Siberia. ¿A qué tipo de identidad nos estamos refiriendo entonces? A priori, este fenómeno rebasa los términos históricos, como recordó hace algunos años en relación a las culturas del Paleolítico Superior en general G.A. Clark (1992).

Tras el análisis historiográfico realizado, lo que parece claro es que la fuerza teórica del particularismo ha sido determinante en la concepción y perdurabilidad del término "Gravetiense", que ha pasado a ser un significante con diferentes significados, las diversas realidades arqueológicas a las que hace referencia. Dicho término se ha convertido en algo tan amplio y genérico que se ha vaciado incluso de su acepción original; recuérdese que ésta era cultural y apelaba, únicamente, a la extensión del utillaje de dorso, las puntas pedunculadas y las venus paleolíticas. Paradójicamente, ha llegado a adoptar un carácter cronológico, como si se tratara estrictamente de un periodo, puesto que algunas de las evidencias arqueológicas que se asociaban al mismo no tenían nada que ver con los ca- 
racteres originales tipológicos que supuestamente lo definieron en un principio y lo perpetuaron posteriormente (Peyrony 1933; Garrod 1936; Sonneville-Bordes 1960; Laplace 1966; Bordes 1968). Por dicho motivo, se han llegado a definir conjuntos culturalmente partiendo simplemente de sus dataciones. A su vez, a veces se encuentran referencias a restos supuestamente "indeterminados" pero de "cronología gravetiense". Actuando de esta manera se está dotando de un nuevo significado al término, convirtiéndolo en una suerte de época o período y así tiene cabida cualquier tipo de variante, puesto que no se apela a unos caracteres específicos industriales o de otra índole (simbólicos, funerarios, etc.), sino a una cronología dada. Si es así como se considera por la mayor parte de la investigación que se debería reformular o discutir su carácter y definición, de manera vaga e imprecisa, en unas ocasiones como período y en otras como macrocultura., entonces la confusión se hace patente o se lleva al extremo cuando se consideran sinónimos tecnocomplejo y período cultural.

Por otra parte, su concepción como tecnocomplejo unitario es también engañosa según se ha visto, puesto que precisamente los modernos estudios de tecnología están parcelando y subdividiendo más y más las evidencias documentadas. Además, resulta altamente restrictivo ceñir las manifestaciones pasadas a la esfera técnica sin relacionarla con otras facetas del registro. En definitiva la práctica tecnológica actual, en ocasiones, parece una mala lectura de los principios teóricos que inspiraron e iniciaron esta corriente, la Paleoetnología de A. Leroi-Gourhan.

Las evidencias que actualmente se incluyen dentro del Gravetiense a escala europea parecen reflejo de ejemplos fascinantes de una notable estratificación social y complejidad a nivel logístico, simbólico y económico, de lo que quizás el caso más emblemático y llamativo sean las evidencias del Pauloviense moravo. Las posibilidades que ofrece el registro gravetiense son mucho más extensas que las teorías que se están proponiendo. Ha habido llamadas de atención en este sentido (Roebroeks et al. 2000), y propuestas que integran diferentes facetas del registro (Soffer 1987, 2000). Es llamativo que dado el peso que ostenta el arte (mueble para el Este y Centro de Europa y parietal y mueble para el Oeste europeo) en este período, no se reivindique desde esta faceta otro tipo de ordenaciones o explicaciones o que muy raramente sea el caso (Fig. 8).

Queremos resaltar finalmente que en esta síntesis no se ha pretendido rechazar las definiciones ni las ordenaciones en Prehistoria, una parte imprescindible de todos los estudios que llevamos a cabo, sino reflexionar sobre cómo se forman, manejan, cambian e interpretan, a través del caso ejemplar del Gravetiense europeo.

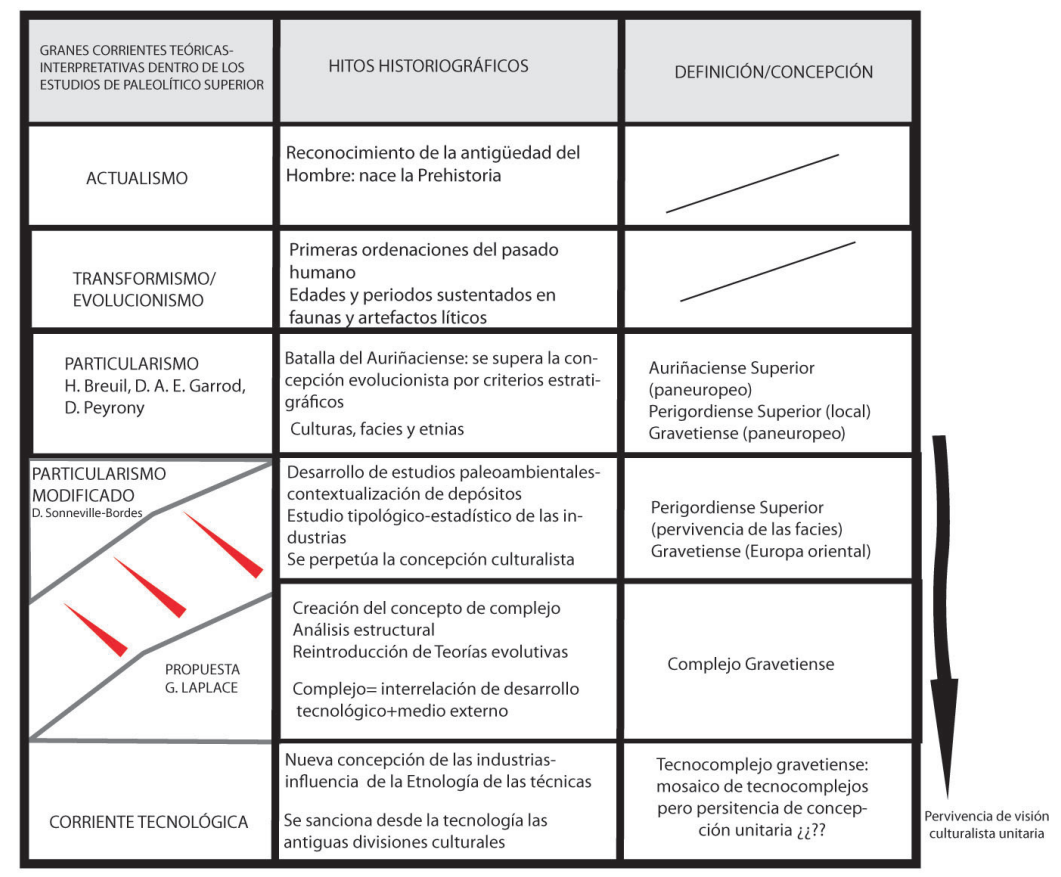

Figura 8.- Visiones del Gravetiense a lo largo del desarrollo de las diferentes propuestas teóricas en Paleolítico Superior del siglo XX. A la izquierda las diferentes corrientes, en el centro los hitos relevantes, a la derecha concepción cultural para cada momento. 


\section{Agradecimientos}

Este texto proviene en origen de una sesión sobre identidad que se organizó con motivo de las II Jornadas de Jóvenes en Investigación Arqueológica (Madrid, mayo de 2009). Agradezco a los organizadores de aquella sesión, Sandra Lozano y Manuel Fernández, que me animaran a participar entonces. Agradezco también la relectura y comentarios de algunos apartados del texto a Adriana Soto, Amaya Mujika, Pilar Alonso y Sarah Hellawell.

\section{Notas}

1. En el marco peninsular también se descubrió la presunta interestratificación Auriñaciense-Perigordiense, en las excavaciones de los años 50 de J. Martínez Santa Olalla en la Cueva del Pendo (niveles VIIIb al V), si bien su publicación fue muy tardía (González Echegaray 1980) y no se puede afirmar que influyera de manera clara en este debate.

2. Esta teoría también se desarrolló en el marco peninsular, a través de la tesis de McCollough (1971), único trabajo específico dedicado al Perigordiense/Gravetiense en este contexto hasta fechas muy recientes.

3. El hallazgo reciente de una Venus paleolítica en un nivel auriñaciense de Hohle Fels quizás sea un indicio de que estas representaciones no son excluyentes de sitios asociados al Gravetiense (Conard 2009).

\section{REFERENCIAS BIBLIOGRÁFICAS}

Almeida, F. (2000): The terminal Gravettian of Portuguese Estremadura: Technological variability of the lithic industries. Dedman College, Southern Methodist University, Dallas.

Arrizabalaga, A. (1995): La industria lítica del Paleolítico Superior inicial en el Oriente cantábrico. Departamento de Geografía, Prehistoria y Arqueología, Universidad del País Vasco (Tesis doctoral inédita).

Aubry, T., Zilhão, J., Almeida, F.; Fontugne, M. (1998): Production d'armatures microlithiques pendant le Paléolithique supérieur et le Mesólithique au Portugal. II Congreso de Arqueología peninsular (R. Balbín, P. Bueno, eds.), Zamora: 259-272.

BINFORD, L. (1973): Interassemblage variability-the Mousterian and the functional argument. The explanation of culture change: models in Prehistory (C. Renfrew, ed.), University of Pittsburg, Pittsburg: 227-254.

Bö̈DA, E. (1989): Le concept Levallois et évaluation de son champ d'application. L'Homme de Néandertal (M. Patou y L. G. Freeman, eds.), Université de Liège, ERAUL, Vol. 4 (La Technique), Lieja: 13-26.

Bon, F. (2002): Les termes de l'Aurignacien. Espacio, Tiempo y Forma, 15, (Serie 1): 39-67.

Bordes, F. (1953): Levalloisien et Moustérien. BSPF, 50, (4): 226-235.

Bordes, F. (1958): Nouvelles Fouilles à Laugerie-Haute Est. Premiers Résultats. l'Anthropologie, 62, (4): 205244.

Bordes, F. (1961): Typologie du Paléolithique Ancien et Moyen. Vol. 2, Imp. Delmas, Burdeos.

Bordes, F. (1968): La question perigordienne. La Préhistoire, problèmes et tendances, C.N.R.S., París: 59-70.

Bordes, F. (1973): On the chronology and the contemporaneity of different Palaeolithic cultures in France. The explanation of culture change: models in Prehistory (C. Renfrew, ed.), University of Pittsburg, Pittsburg: 217226.

Bordes, F.; Sonneville-Bordes, D. (1970): The significance of variability in Palaeolithic assemblages. World Archaeology, 1: 61-76.

Breuil, H. (1906): Les gisements présolutréens du type d'Aurignac. Coup d'œil sur le plus ancien âge du Renne. Extrait du Compte Rendu du XIII Congrès d'Anthropologie et d'Archéologie Préhistoriques, Mónaco.

Breuil, H. (1907): La question aurignacienne. Étude critique de stratigraphie comparée. Extrait de la Revue préhistorique. 2nd Année, no 6 et 7: 173-219.

Breuil, H. (1909): L'Aurignacien présolutréen. Epilogue d'une controverse. Extrait de la Revue préhistorique. 4e Année, 8 et 9: 229-248 y 265-286.

Breuil, H. (1912): Les subdivisions du Paléolithique Supérieur et leur signification. Congrés International d'Archéologie préhistorique. Compte Rendu de la XIV session, Géneve.

Breuil, H.; Lantier, R. (1959): Les hommes de la pierre ancienne. Payot, París. 
Bricker, H. M. (1995): Le Paléolithique supérieur de l'Abri Pataud (Dordogne): Les fouilles de H. L. Movius Jr. Documents d' archéologie française, vol. 50, París.

Cahen, D., Karlin, C., Keeley, L. H.; Van Noten, F. (1981): Méthodes d'analyse technique, spatiale et fonctionnelle d'ensembles lithiques. Hellinium, 20: 209-259.

Clark, G. A. (1992): La migración como una no-explicación en la arqueología paleolítica. Elefantes, ciervos y ovicaprinos (Moure, A., ed.), Universidad de Cantabria, Santander: 17-36.

Clarke, D. L. (1968): Analytical Archaeology. Methuen, Londres.

Champagne, F.; Espitalie, R. (1967): La stratigraphie du gisement du Piage: note préliminaire. BSPF, 64, (1): 2934.

Cheynier, A. (1960): Place pour le Gravétien. BSPF, 57, (7-8): 389-412.

Conard, N. J. (2009): A female figurine from the basal Aurignacian of Hohle Fels Cave in Southwestern Germany. Nature, 459: 248-252.

DAvid, N. (1973): On upper Palaeolithic society, ecology and technological change: the Noaillian case. The explanation of culture change: models in Prehistory (C. Renfrew, ed.), University of Pittsburgh Press, Pittsburgh: 267-303.

David, N. (1985): The Noaillian (level 4) assemblages and the Noaillian culture in Western Europe. American School of prehistoric research, 37, Cambridge.

David, N.; Bricker, H. M. (1987): Perigordian and Noaillian in the Greater Perigord. The Pleistocene Old World. Regional Perspectives (Soffer, O., ed.), Plenum, Nueva York: 237-250.

Davies, W. (1999): Dorothy Annie Elisabeth Garrod (5th May,1892-18th December, 1968). Dorothy Garrod and the Progress of the Palaeolithic (W. Davies, R. Charles, eds.), Oxbow books, Oxford: 1-14.

Delporte, H. (1954): Le Perigordien. Le grandes civilisations préhistoriques de la France. Livre jubilaire de la Société Préhistorique française (1904-1954), LI: 44-48.

Delporte, H. (1982): La imagen de la mujer en el Arte Prehistórico. Istmo, Madrid.

Demoule, J.-P. (2004): André Leroi-Gourhan, l'ethnie, la culture et le préhistorien: historie d'un rendez-vous manqué. Autour de l'homme: contexte et actualité d'André Leroi-Gourhan (F. Audouze, N. Schalanger, eds.), APDCA, Antibes: 45-68.

Duinduan, F. (1999): Chronologie du peuplement gravettien sur les côtes de Méditerranée occidentale. Les faciès leptolithiques du Nord-Ouest Méditerranéen: Milieux naturels et culturels. XXIV Congres Préhistorique de France (D. Sacchi, ed.), Societé Préhistorique Française, Carcassone: 127-138.

Dindjian, F. (2002): Cinquante années de recherches sur les débuts de l'Aurignacien en Europe occidentale. Espacio, Tiempo y Forma, 15: 17-38.

Dindjian, F.; Bosselin, B. (1994): Périgordien et Gravettien: L'Epilogue d'une contradiction? Prehistoire Européenne, 6: 117-131.

Dindjian, F., Kozlowski, J. K. y Otte, M. (1999): Le Paléolithique supérieur en Europe. Armand Colin, París.

Einwögerer, T., Friesinger, H., Händel, M., Neugebauer-Maresch, C., Simon, U.; Teschler-Nicola, M. (2006): Upper Palaeolithic infant burials. Nature, 444: 285.

Foucher, P. (2005): Gargas et l'Atlantique: les relations transpyrénéennes au cours du Gravettien. Munibe, 52, (2): 131-147.

Fullola, J. M., Roman, D., Soler, N. y Villaverde, V. (2007): Le Gravettien de la côte méditerranéenne ibérique. Paléo, 19: 73-88.

Gamble, C. (1982): Interaction and alliance in Palaeolithic society. Man, 17, (1): 92-107.

Gamble, C. (2001): Las sociedades paleoliticas de Europa. Ariel, Barcelona.

Garrod, D. A. E. (1936): The Upper Palaeolithic in the light of recent discoveries. Nature: 826-830.

GeERTz, C. (2005): Descripción densa: hacia una teoría interpretativa de la cultura. La interpretación de las culturas, Gedisa, Barcelona: 19-40.

GonzÁlez Echegaray, J. (1980): El yacimiento de la Cueva de El Pendo (excavaciones 1953-57). Bibliotheca Praehistorica Hispana, Consejo Superior de Investigaciones Científicas, Instituto Español de Prehistoria, Vol. XVII, Madrid.

Groenen, M. (1994): Pour une histoire de la préhistoire. Le Paléolithique. Jérôme Millon, Grenoble. 
Guillermin, P. (2004): Réflexions sur l'interprétation des industries gravettiennes à partir de l'étude typo-technologique d'une occupation spécialisée: la couche E du gisement des Fieux (Miers, Lot). Memoria de DEA, Université de Toulouse Le Mirail.

Henry-Gambier, D. (2005): Évolutions des pratiques funéraires en Italie au Paléolithique supérieur. Comportements des hommes du Paléolithique moyen et supérieur en Europe: territoires et milieux. Actes du Colloque du G. D. R. (D. Vialou, J. Renault-Miskovsky, M. Patou-Mathis eds.), Vol. 111, ERAUL, Lieja: 213-229.

Henry-Gambier, D., Bruzek, J., Schmitt, A., Houët, F.; Murail, P. (2006): Révision du sexe et de l'âge au décès des fossiles de Cro-Magnon (Dordogne, France) à partir de l'os coxal. Palevol, 5: 735-741.

Higham, T. F. G., Jacobi, R. M., Basell, L. S., Bronk, C., Chiotti, L. y Nespoulet, R. (2011): Precision dating of the Palaeolithic: A new radiocarbon chronology for the Abri Pataud (France), a key Aurignacian sequence. Journal of Human Evolution, 61(5): 1-15.

Johnson, M. (2010): Archaeological theory. An Introduction. Wiley-Blackwell, Oxford.

JordÁ, F. (1954): Gravetiense y Epigravetiense en la España Mediterránea. Caesaraugusta, 4: 7-30.

Karlin, C., Bodu, P. y Pelegrin, J. (1991): Processus techniques et chaînes opératoires. Comment les préhistoriens s'approprient un concept élaboré par les ethnologues. Observer l'action technique, les chaînes opératoires, pour quoi faire? (H. Balfet, ed.), CNRS, París: 101-117.

KLARIC, L. (2003): L'unité technique des industries a burin du Raysse dans leur contexte diachronique. Reflexions sur la diversite culturelle au gravettien a partir des donnes de la Picardie, d'Arcy-Sur-Cure, de Brassempouy et du cirque de la Patrie. U. F. R. D'Histoire de l'art et d'archéologie. Tesis doctoral inédita, Université Paris I.

Klaric, L., Guillermin, P. y Aubry, T. (2009): Des armatures variées et des modes de productions variables. Réflexions à partir de quelques exemples issus du Gravettien d'Europe occidentales (France, Portugal, Allemagne). Gallia Préhistoire, 51: 113-154.

Kozlowski, J. K. (2005): Paléolithique supérieur et Mésolithique en Méditerranée: cadre culturel. l'Anthropologie, 109: 520-540.

LAPlACE, G. (1956): Typologie statistique et évolution des complexes à lames et lamelles. BSPF, 53, (5-6): 271290.

LAPlace, G. (1966): Recherches sur l'origine et l'évolution des complexes leptolithiques. Vol. 4, École Française de Rome, Mélanges d'Archéologie et d'Histoire, París.

LAPlace, G. (1972): La Typologie analytique et structurale: base rationnelle d'étude des industries lithiques et osseuses. Colloques nationaux du centre National de la Recherche Scientifique, Banques de donnes archéologiques, 932, Marsella.

Laville, H.; Rigaud, J.-P. (1973): The Perigordian V industries in Périgord: typological variations, stratigraphy and relative chronology. World Archaeology, 4: 330-338.

Lemonnier, P. (1991): De la culture matérielle à la culture? Ethnologie des techniques et Préhistoire. 25 Ans d'études technologiques en Préhistoire. XI Rencontres Internationales d'Archeologie et d'Histoire d'Antibes, APDCA, Antibes: 15-20.

LENOIR, M. (1974): Faciès et culture. BSPF, 71, (2): 58-64.

Leroi-Gourhan, A. (1961): Les fouilles d'Arcy-sur-Cure. Gallia Préhistoire, 4: 3-16.

Leroi-Gourhan, A. (1989a): El Medio y la Técnica I. Taurus, Madrid.

Leroi-Gourhan, A. (1989b): El Medio y la Técnica II. Taurus, Madrid.

Leroi-Gourhan, A.; Brezillon, M. (1972): Fouilles de Pincevent. Essai d'analyse ethnographique d'un habitat magdalénien. Gallia Préhistoire, VII (Suplement).

Leroi-Gourhan, A. (ed.) (1976): Les structures d'habitat au Paléolithique supérieur. Colloque XIII Union Internationale des Sciences Préhistoriques et Protohistoriques. CNRS, París.

McCollough, M. R. C. (1971): Perigordian facies in the Upper Palaeolithic of Cantabria. University of Pennsylvania (Tesis doctoral inédita).

Mellars, P. (1973): The character of the Middle-Upper Palaeolithic transition in south-west France. The explanation of culture change: models in Prehistory (C. Renfrew, ed.), University of Pittsgburgh Press.

Moreau, L. (2010): Geißeklösterle. The Swabian Gravettian in its European context. Quartär, 57: 79-93.

Mortillet, G. (1885): Le Préhistorique. Antiquité de l'Homme (Deuxième Édition). C. Reinwald, París.

Moure, A. y GonzÁlez, M. G. (1984-1999): Excavaciones y documentación del arte rupestre de la Cueva de la Fuente del Salín (Muñorrodero, Val de San Vicente). Actuaciones arqueológicas en Cantabria. Santander. 
Movius, H. L. (1977): Excavation of the Abri Pataud, Les Eyzies. Stratigraphy. American School of Prehistoric Research Bulletin, 31, Cambridge Mass.:1-167.

Mussi, M., Cinq-Mars, J. y Bolduc, P. (2000): Echoes from the mammoth steppe: The case of Balzi Rossi. Hunthers of the Golden Age (W. Roebroeks, M. Mussi, J. Svoboda, K. Fennema, eds.), University of Leiden, Leiden: 105-124.

Nespoulet, R. (1995): Le Périgordien VI de l'abri Pataud, les Eyzies-de-Tayac, Dordogne. Etude technologique et typologique de l'industrie lithique de la couche 3. Tesis doctoral, Institut de Paléontologie Humaine, Muséum National d'Histoire Naturelle.

Norret, P. (2007): Le Gravettien de Moldavie (30000-23000 BP). Páleo, 19: 159-180.

Oliva, M. (2000): Some thoughts on paulovian adaptations and their alternatives. Hunters of the Golden Age. The Mid-Upper Paleolithic of Eurasia 30000-20000BP (W. Roebroeks, M. Mussi, J. Svoboda, K. Fennema, eds.), University of Leiden, Leiden: 219-229.

Oliva, M. (2004): Les cabanes en os de mammouth du Paulovien morave. Mythe o réalité? Dossiers d'Archeologie, 291: $52-55$

Onoratini, G.; Raux, A. (1992): Les cultures du Paléolithique Supérieur ancien de Provence Orientale. Bulletin du Musée d'anthropologie préhistorique de Monaco, 35: 65-114.

Отте, M. (1985): Le Gravettien en Europe. L'Anthropologie, 89: 479-503.

Отте, M. (2005): Territoires et styles. Comportements des hommes du Paléolithique moyen et supérieur en Europe: territoires et milieux. Actes du Colloque du G. D. R. (D. Vialou, J. Renault-Miskovsky, M. Patou-Mathis, eds.), Vol. 111, ERAUL, Lieja: 157-161.

Otte, M. y Keeley, L. H. (1990): The impact of regionalism on Paleolithic studies. Current Anthropology, 31, (5): 577-582.

Palma Di Cesnola, A. (2001): Le Paléolithique supérieur en Italie. Jerôme Million, París.

Pelegrin, J. (1990): Prehistoric lithic technology: some aspects of research. Archaeological Review from Cambridge, 9, (1): 116-125.

Pelegrin, J. (1995): Technologie lithique. Le Châtelperronien de Roc-De-Combe (Lot) et de la Côte (Dordogne). Vol. 20, Cahiers du Quaternaire, París.

PeÑa, P. DE LA (2011): Sobre la unidad tecnológica del Gravetiense en la Península Ibérica: implicaciones para el conocimiento del Paleolitico Superior inicial. Departamento de Prehistoria, Universidad Complutense de Madrid (Tesis doctoral inédita).

PERLÈs, C. (1992): In Search of Lithic Strategies. A cognitive approach to prehistoric chipped stone assemblages. Representations in Archaeology (J.-C. Gardin, S. Peebles, eds.), Indiana University Press, Indiana: 223-247.

Perrineau, P. (1975): Sur la notion de culture en anthropologie. Revue française de science politique, 5: $946-968$.

Pesesse, D. (2010): Quelques repères pour mieux comprendre l'émergence du Gravettien en France. BSPF, 107, (3): 465-487.

Pétrequin, P. y Pétrequin, A.-M. (2002): Écologie d'un outil: la hache de la pierre en Irian Jaya (Indonésie). CNRS, París.

Peyrony, D. (1933): Les industries aurignaciennes dans le bassin de le Vézère. BSPF: 543-559.

Peyrony, D. (1936): Le Périgordien et l'Aurignacien. BSPF, T. XXXIII, (11): 616-619.

Peyrony, D. (1946): Une mise au point au sujet de l'Aurignacien et du Périgordien. BSPF: 232-237.

Pigeot, N. (1987): Magdaléniens d'Etiolles. Économie de débitage et organisation sociale (L'unité d'habitat U5). Supplément à Gallia Préhistoire, Éditions du CNRS, XXV, París.

Pigeot, N. D. (2004): Les derniers magdaléniens d'Etiolles. Éditions du CNRS, París.

Pottier, C. (2005): Le Gravettien moyen de l'abri Pataud (Dordogne, France): le niveau 4 et l'eboulis 3/4. Etude technologique et typologique de l'industrie lithique. Museum National d'Histoire Naturelle. Département de Préhistoire, Tesis doctoral.

Ripoll, S., Baldellou, V., Muñoz, F. J. y Ayuso, P. (2001): La Fuente del Trucho (Aque-Colungo, Huesca). Bolskan, 18, (211-224).

Ripoll, S., Ripoll, E. y Collado, H. (1999): Maltravieso. El Santuario extremeño de las manos. Memorias, Junta de Extremadura, Consejería de Cultura-Museo de Cáceres, 1, Mérida.

Roebroeks, W.; Corbey, R. (2000): Periodisations and double standards in the study of the Palaeolithic. Hunters of the Golden Age (W. Roebroeks, M. Mussi, J. Svoboda, K. Fennema, eds.), University of Leiden, Leiden: 77-86. 
Roebroeks, W., Mussi, M., Svoboda, J. y Fennema, K. (Eds.) (2000): Hunters of the Golden Age. The Mid-Upper Palaeolithic of Eurasia 30000-20000BP. University of Leiden, Leiden.

Simonet, A. (2009a): Les gravettiens des Pyrénées. Des armes aux sociétés. Tesis doctoral, Université de Toulouse-Le Mirail.

Simonet, A. (2009b): Les gravettiens des Pyrénées. Des armes aux sociétés. Munibe, 60: 81-98.

Soffer, O. (1987): Upper Paleolithic Connubia, Refugia, and the archaeological Record from Eastern Europe. The Pleistocene of the Old World. Regional Perspectives (O. Soffer, ed.), New York: 333-348.

Soffer, O. (2000): Gravettians technologies in social contexts. Hunters of the Golden Age (W. Roebroeks, M. Mussi, J. Svoboda, K. Fennema, eds.), University of Leiden, Leiden: 59-75.

Sonneville-Bordes, D. (1955): La question du Périgordien II. BSPF, 52, (3-4): 187-200.

Sonneville-Bordes, D. (1960): Recherches sur le Paléolithique Supérieur en Périgord. Université de Paris.

Sonneville-Bordes, D. (1966): L'Evolution du Paléolithique supérieur en Europe Occidentale et sa signification. BSPF, LXIII, (1): 3-34.

Sonneville-Bordes, D.; Perrot, J. (1953): Essai d'adaptation des méthodes statistiques au Paléolithique supérieur. Premiers résultats. $B S P F: 323-333$.

Sonneville-Bordes, D. y Perrot, J. (1954): Lexique typologique du Paléolithique supérieur. BSPF, I, (7): $327-335$.

Sonneville-Bordes, D.; Perrot, J. (1955a): Lexique typologique du Paléolithique supérieur. BSPF, II, (1): 76-79.

Sonneville-Bordes, D. (1955b): À propos du Périgordien. BSPF, 52, (9-10): 597-601.

Sonneville-Bordes, D.; Perrot, J. (1956): Lexique typologique du Paléolithique supérieur. BSPF, III, (7-8-9).

Svoboda, J. (1994): The Paulov site, Czech Republic: Lithic evidence from the Upper Paleolithic. Journal of Field Archaeology, 21, (1): 69-81.

Svoboda, J. (2007): The Gravettian on the Middle Danube. Páleo, 19, (209-220).

Svoboda, J., Klíma, B., Jarosová, L.; Skrdla, P. (2000): The Gravettian in Moravia: climate, behaviour and technological complexity. Hunters of the Golden Age (W. Roebroeks, M. Mussi, J. Svoboda, K. Fennema, eds.), University of Leiden, Leiden: 197-217.

Tostevin, G. B. (en prensa): Levels of theory and social practice in the reduction sequence and Chaîne Opératoire methods of lithic analysis.

Tylor, E. B. (1871): Primitive Culture. John Murray, Londres.

VASIL'Ev, S. (2000): The Siberian mosaic: Upper paleolithic adaptations and change before last Glacial Maximum. Hunters of the Golden Age (W. Roebroeks, M. Mussi, J. Svoboda, K. Fennema, eds.), University of Leiden, Leiden: 173-196.

Vega Toscano, L. G. (1988): El Paleolitico Medio del sureste español y Andalucía Oriental. Tesis doctoral inédita, Departamento de Prehistoria, Universidad Complutense de Madrid.

Vega Toscano, L. G. (2001): Aplicación de la metodología de los programas de investigación al análisis historiográfico del Paleolítico. Complutum, 12: 185-215.

Vicent, J. M. (1982): Las tendencias metodológicas en Prehistoria. Trabajos de Prehistoria, 39: 9-53.

VicEnT, J. M. (1985): Un concepto de metodología. Hacia una definición epistemológica de prehistoria y Arqueología. Actas de II Jornadas de Metodología y Didáctica de la Historia (Prehistoria y Arqueología), Universidad de Extremadura, Cáceres: 55-72.

VV.AA. (2006): Towards a definition of the Aurignacian. Proceedings of the Symposium held in Lisbon, Portugal, June 25-30, 2002. Vol. 45, Trabalhos de Arqueologia, Lisboa.

Watson, P. J. (1995): Archaeology, Anthropology and the Culture Concept. American Anthropologist, 97, (4): 683-694.

White, L. (1959): The Evolution of Culture. McGraw-Hill, Nueva York.

ZıLhão, J. (1997): O Paleolitico Superior da Estremadura Portuguesa. Ed. Colibri, Lisboa.

ZıLHÃo, J. (2005): Burial evidence for the social differentiation of age classes in the Early Upper Palaeolithic. Comportements des hommes du Paléolithique moyen et supérieur en Europe: territoires et milieux. Actes du Colloque $d u$ G. D. R. (D. Vialou, M. Renault-Miskovsky, M. Patou-Mathis, eds.), Vol. 111, ERAUL, Lieja: 231-241.

ZvelebIL, M. (2005): Zvelebil, M. (2009): The Mesolithic and the 21st Century. Mesolithic Horizons (McCartan, S. B., Schulting, R. J., Warren, G. y Woodman, P., eds.), Papers presented at the 7th International conference on the Mesolithic in Europe, Belfast, 2005, vol. I: xlvii-lviii. Oxford, Oxbow Books. 